\title{
Upscaling of Transport Processes in Porous Media with Biofilms in Non-Equilibrium Conditions
}

\author{
Laurent Orgogozo $^{1}$, Fabrice Golfier ${ }^{1}$, Michel Buès ${ }^{1}$, Michel Quintard ${ }^{2}$ \\ ${ }^{1}$ Laboratoire Environnement, Géomécanique et Ouvrages, Nancy-Université, Rue du Doyen Roubault - BP 40 F - \\ 54501 Vandœuvre-lès-Nancy, France \\ ${ }^{2}$ Institut de Mécanique des Fluides de Toulouse, Allée du Professeur Camille Soula, 31400 Toulouse, France \\ * Corresponding author. Tel.: (33) 03835963 01; Fax: (33) 0383596300. \\ E-mail address: Laurent.Orgogozo@ensg.inpl-nancy.fr
}

\begin{abstract}
In this work, we derive two different Darcy-scale models for the transport of biodegradable solutes in porous media containing a microbial biomass that developed under the form of a biofilm. Biofilms are composed of bacterial populations and extra cellular polymeric substances, and grow attached to a solid-fluid interface, e.g. the pore walls of a water-saturated porous medium. We begin with the pore-scale description of mass transport, mass transfer between phases (fluid phase - generally water - and biofilm phase) and biologically-mediated reactions. Then, two limit cases of non-equilibrium transport are identified and characterized. Finally, these processes are upscaled using the method of volume averaging to obtain two different macroscale mass balance models. The models are derived for specific cases of non-equilibrium reactive transport (i.e., spatial concentration gradients may exist in one or both phases), for which mechanisms of mass transfer or reaction kinetics limit the rate of biodegradation. In both cases, a
\end{abstract}


one-equation model can be developed even if non-equilibrium conditions exist. The validity domains of the two considered transport models (the Reaction Rate Limited Consumption model

- RRLC model - and the Mass Transfer Limited Consumption model - MTLC model) are established in terms of reactive and hydrodynamic conditions of transport (Damköhler number and Péclet number). The RRLC model is found to be consistent with direct numerical simulation (DNS) results at high Péclet and Damköhler numbers, while the applicability of the MTLC model is limited to high Damköhler numbers but low Péclet numbers.

\section{Keywords}

Porous media; biofilm; biodegradation; reactive transport; non-equilibrium; upscaling.

\section{Introduction}

Porous-medium-supported biofilms have been used to treat contaminants for well over one hundred years (e.g.: the use of trickling filters began in the UK in the late 1890s and in the US in 1901). Since these early uses, porous-medium-supported biofilms have proved to be useful in a number of applications from the highly engineered (e.g., immobilized cell reactors, anaerobic packed beds, eukaryotic cell propagation) to the more empirically operated (e.g., air filtration in composting biofilters). In subsurface systems, biofilms are naturally present in both the saturated and unsaturated zones. Such biofilms can degrade contaminants in both passive (natural attenuation) and actively-modified systems. This idea has led to the development of in situ bioremediation methods (e.g. [32], [28]). There is an extensive literature on this subject, and a number of review articles summarize the current state-of-the-art methods for subsurface bioremediation (e.g. [3], [10], [42], [45], [47], [52], and [53]).

One of the challenging features in the development of models for groundwater 
bioremediation processes (or for bioprocesses in chemical engineering) is the multiscale aspect of solutes in porous media, from the cell scale to the application scale (the field scale or the reactor scale). Intermediate levels of upscaling could be interjected into this conceptual scheme, if additional heterogeneities are to be taken into account(e.g., if the biofilm porosity or additional levels of geological heterogeneities need to be considered). Although in principle it should make it ideal to solve a model of a large-scale bioreactor (Figure 1, Level IV) at the smallest length scale of interest (e.g., the characteristic length associated with the structure of the cell scale) this is generally impractical because (1) the full microscale data is generally not available, and (2) the resulting model would contain far more detail (i.e., information) than would be useful for practical applications, which can be a serious drawback in terms of data storage and computation time. One solution to this problem is to develop formal macroscale models that replace the microscale details of the system by effective representations. Generally, these effective descriptions are carried out by identifying a scaling law that allows one to honor some (usually statistical) aggregate measures of the microscale phenomena (cf. [62]). In this work, we adopt a volume averaging method [55] in order to filter information from a smaller length scale to a larger one and, eventually, to the length scale at which the system analysis takes place. The scaling law used in this analysis is the assumption of separation of scales (e.g. [55]) between the micro- and macro-scales of interest. For the configuration illustrated above one can theoretically evaluate Darcy scale parameters in a relevant way by using successively two times this method (for field scale or reactor scale parameters additional upscalings may be necessary to take into account the large scale heterogeneities such as fractures, high-permeability lenses, ...).

The averaging of the processes which take place at the smallest length scale associated with this problem (i.e., averaging from the cell scale - Figure 1, level I - to the biofilm scale - Figure 1, level II - , at which the biofilm is considered as a continuous and homogeneous phase) has been 
previously examined ([57], [58], [59], [60]). In this work, we start with these previous results to

perform the second averaging in this hierarchy (boxed in red in Figure 1). The formalism of volume averaging should help to define exactly how the Darcy scale effective parameters are linked to the pore and biofilm scale processes. Figure 2 details the characteristic lengths of the pore scale at which is defined the volume of averaging considered in this work, denoted $V$. We start with this description and we upscale from this scale (Figure 1, level II, with a characteristic length $\ell_{\gamma}$ or $\ell_{\omega}$ ) to the Darcy-scale (Figure 1, level III, with a characteristic length $L$ ). Note here that the above-mentioned assumption of separation of scales - required for such an upscaling specifies that the characteristic length of the averaging volume, $R_{0}$, should be very large compared to $\ell_{\gamma}$ and $\ell_{\omega}$ and very small relative to $L$. Several studies have been conducted that develop a one-equation macroscopic transport model for the conditions of local mass equilibrium ([18], [61]), i.e. in the case of equilibrium between both phases with respect to mass transfer processes. This model requires that the averaged concentration changes over characteristic lengths that are large relative to the characteristic length associated with the averaging volume. The model obtained by these studies is similar to the model that is often heuristically posed for biological processes in porous media; however, the averaging process allows one to carefully define the domain of validity for the equilibrium assumption [18].

Under non-equilibrium conditions, two one-equation macroscopic transport models can also be considered and this is the focus of this paper. The first model is based on the assumption that the characteristic time for mass transfer from the fluid phase to the biofilm phase is smaller than the characteristic time for microbial kinetic reactions in the biofilm phase. This model has been explored extensively in a number of classical works that represent the reaction rate limitation by an effectiveness factor (e.g. [2]), and it corresponds to the shallow mass transfer gradient case in the terminology of Rittmann and McCarty [43]. For this work, we will refer to it as the reaction-rate-limited-consumption (RRLC) case. The second, hereafter referred to as Mass 
Transfer Limited Consumption (MTLC) model, has also been considered in the literature ([22], [29], [56]) and assumes that the exchange mass rate between the two regions is slow relative to the biodegradation rate in the biofilm phase. There are numerous examples ([27], [31], [12]), indeed, in groundwater systems or in bioengineered reactors for which external (relatively to the reactive phase, here the biofilm phase) mass transfer is the limiting step of microbial processes (e.g., biofilm growth under oxygen-limiting conditions).

This paper is organized as follows: in section 2 we present the considered pore-scale transport model which is based partly on the previous upscaling work (from level I to level II in Figure 1) performed by Wood and Whitaker ([57], [59]). In section 3, we detail in which manner the two one-equation non-equilibrium models can be derived from the general two-equation model. In section 4 and section 5, the Reaction Rate Limited Consumption model and the Mass Transfer Limited Consumption model are developed, respectively. In section 6, we will present some numerical validations of the models, and establish the domain of validity associated to both models. In section 7, we will present the conclusions and perspectives related to this theoretical development.

\section{Pore-scale model}

The basis of this investigation is the set of mass balance equations which are used for describing mass conservation at the pore scale (Level II in Figure 1) in the fluid (water; $\gamma$-phase) and the biofilm (polymeric gel containing bacterial populations which develops on the interface fluid-solid; denoted as the $\omega$-phase). In the $\omega$-phase, only diffusion and reaction phenomena take place, while in the $\gamma$-phase, the transport phenomena are convection and diffusion. For this analysis, we have focused on systems with a single substrate (carbon and energy source - species $A$ ) and a single electron acceptor (such as dioxygen - species $B$ ) and have assumed a classical 
dual-Monod form for the kinetics of biodegradation [30]. For this work we do not consider the

production of biomass associated with the substrate consumption (nor the biomass decrease due to decay or detachment), because we have made the classical approximation that the time scale associated with changes in the biomass phase is much larger than the time scale associated with the transport and the consumption of the substrate (generally, at least two orders of magnitude difference [34]). The mass balance equations for solute transport and biofilm growth are thus uncoupled. The solid phase ( $\kappa$-phase) is assumed to be impermeable to all chemical species. More details about the development of this set of equations can be found in Golfier et al. [18]). Under these assumptions, the mass balance equations for the fluid-biofilm-solid system can be written as follows:

$\omega$-phase

$$
\frac{\partial c_{A \omega}}{\partial t}=\nabla \cdot\left(\mathbf{D}_{A \omega} \cdot \nabla c_{A \omega}\right)-\mu_{A \omega} \rho_{\omega} \frac{c_{A \omega}}{c_{A \omega}+K_{A \omega}} \frac{c_{B \omega}}{c_{B \omega}+K_{B \omega}} \quad \text { in the } \omega \text {-phase, }
$$

$\gamma$-phase

$$
\frac{\partial c_{A \gamma}}{\partial t}+\nabla \cdot\left(\mathbf{v}_{\gamma} c_{A \gamma}\right)=\nabla \cdot\left(\mathcal{D}_{A \gamma} \nabla c_{A \gamma}\right) \quad \text { in the } \gamma \text {-phase }
$$

B.C.1

$$
\mathbf{n}_{\omega \kappa} \cdot \mathbf{D}_{A \omega} \cdot \nabla c_{A \omega}=0 \text { at } A_{\omega \kappa},
$$

B.C. 2

$$
\mathbf{n}_{\gamma \kappa} \cdot \mathcal{D}_{A \gamma} \nabla c_{A \gamma}=0, \quad \text { at } A_{\gamma \kappa}
$$

B.C.3

$$
c_{A \gamma}=K_{A, e q} c_{A \omega}, \text { at } A_{\gamma \omega}
$$

B.C.4

$$
\mathbf{n}_{\gamma \omega} \cdot \mathbf{D}_{A \omega} \cdot \nabla c_{A \omega}=\mathbf{n}_{\gamma \omega} \cdot \mathcal{D}_{A \gamma} \nabla c_{A \gamma} \text { at } A_{\gamma \omega},
$$

Here, $c_{A \gamma}$ and $c_{A \omega}$ represent the concentration of species $A$ (substrate, generally an organic solute) in the $\gamma$ and $\omega$-phases, respectively $\left[\mathrm{mol} . \mathrm{m}^{-3}\right] ; \mathcal{D}_{A \gamma}$ is the diffusion coefficient of species $A$ in the fluid and $\mathbf{D}_{A \omega}$ is the effective diffusion tensor for species $A$ in the biofilm (its tensorial nature is due to the fact that the biofilm phase is not necessarily homogeneous and isotropic; see [57] for 
more details on this tensor) $\left[\mathrm{m}^{2} \cdot \mathrm{s}^{-1}\right] ; \mathbf{v}_{\gamma}$ is the fluid velocity $\left[\mathrm{m} \cdot \mathrm{s}^{-1}\right] ; K_{A, e q}$ is the equilibrium partition coefficient between the $\gamma$ and $\omega$-phases $[-] ; \mu_{A \omega}$ is the specific degradation rate for the substrate $\left[\mathrm{s}^{-1}\right] ; \rho_{\omega}$ is the microbial density $\left[\mathrm{mol} . \mathrm{m}^{-3}\right] ; K_{A \omega}$ and $K_{B \omega}$ are the half-saturation constants for the substrate and electron acceptor, respectively $\left[\mathrm{mol} . \mathrm{m}^{-3}\right]$; and $c_{B \omega}$ is the concentration of electron acceptor in the $\omega$-phase $\left[\mathrm{mol} \cdot \mathrm{m}^{-3}\right]$. We have used the terminology $A_{\gamma \kappa}$ to indicate the interface between the $\gamma$ - and $\kappa$ phases and $A_{\gamma \omega}$ and $A_{\omega \kappa}$ for the interface between the $\gamma$ - and $\omega$-phases and between the $\kappa$ - and $\omega$-phases, respectively. The term $\mathbf{n}_{\gamma \kappa}$ represents the unit normal pointing outward from the $\gamma$-phase toward the $\kappa$-phase; $\mathbf{n}_{\gamma \omega}$ and $\mathbf{n}_{\omega \kappa}$ are similarly defined. Note that a same set of mass balance equations also holds for species $B$, but because the analysis is identical to that for species $A$, we will not explicitly derive them here. These two sets of mass balance equations are coupled through the dual Monod kinetics of the reaction terms. In this work, for the simplicity of the exposure, we will make the assumption that species B is in large excess, so that $c_{B \omega}$ could be considered as temporally and spatially constant. The resulting system of governing equations depends only on species A. Taking into account the coupling between the transport of species A and B is possible in the framework developed below, and it will be the subject of a further study.

\section{Two limit cases leading to the development of simplified non equilibrium models}

\subsection{Unclosed form of the averaged equations}

Referring to the averaging volume, $V$, illustrated in Figure 1, we define the superficial average concentration of species $A$ in the $\omega$-phase (biofilm) as: 


$$
\left\langle c_{A \omega}\right\rangle=\frac{1}{V} \int_{V_{\omega}} c_{A \omega} d V
$$

Here $V_{\omega}$ represents the volume of the $\omega$-phase contained in the averaging volume. A similar definition holds for the average concentration of species $A$ in the $\gamma$-phase:

$$
\left\langle c_{A \gamma}\right\rangle=\frac{1}{V} \int_{V_{\gamma}} c_{A \gamma} d V
$$

In applications it is often preferable to work with a concentration that represents the average over the fluid phase (i.e., the pore space in a saturated porous medium) rather than the total volume of the porous medium. This concentration is the intrinsic average concentration of the $\gamma$-phase. An intrinsic average concentration can also be defined in the $\omega$-phase; these concentrations are defined as follows:

$$
\begin{aligned}
\left\langle c_{A \omega}\right\rangle^{\omega} & =\frac{1}{V_{\omega}} \int_{V_{\omega}} c_{A \omega} d V \\
\left\langle c_{A \gamma}\right\rangle^{\gamma} & =\frac{1}{V_{\gamma}} \int_{V_{\gamma}} c_{A \gamma} d V
\end{aligned}
$$

One can note that the intrinsic and superficial averages are related through the volume fractions of the two phases:

$$
\begin{aligned}
& \left\langle c_{A \omega}\right\rangle=\varepsilon_{\omega}\left\langle c_{A \omega}\right\rangle^{\omega} \\
& \left\langle c_{A \gamma}\right\rangle=\varepsilon_{\gamma}\left\langle c_{A \gamma}\right\rangle^{\gamma}
\end{aligned}
$$

where $\varepsilon_{\omega}$ and $\varepsilon_{\gamma}$ represent the volume fractions of the $\omega$ - and $\gamma$-phases respectively. Since we supposed that the time scale associated with the biomass production is much larger than the time scale associated with the transport and the consumption of the substrate, these volume fractions are treated as constants in time.

In the following development, in order to remove averaged quantities from point values, we will make use of Gray's decompositions [19] for the concentration fields: 


$$
c_{A \omega}=\left\langle c_{A \omega}\right\rangle^{\omega}+\tilde{c}_{A \omega}
$$

$$
c_{A \gamma}=\left\langle c_{A \gamma}\right\rangle^{\gamma}+\tilde{c}_{A \gamma}
$$

The quantities $\tilde{c}_{A \omega}$ and $\tilde{c}_{A \gamma}$ are referred to as spatial deviation concentrations.

The process of volume averaging is initiated by forming the superficial average of the phase conservation equations, (1) to (6). In order to interchange time derivative and averaging operators, one must make use of the general transport theorem [54], while for spatial derivative, the spatial averaging theorem ([19], [21]) is required. The complete development for this first step in averaging equations (1) to (6) is available in Golfier et al. ([18], Appendix A), and the result is: Averaged equation for the biofilm ( $\omega$-phase)

$$
\begin{aligned}
& \underbrace{\frac{\partial\left(\varepsilon_{\omega}\left\langle c_{A \omega}\right\rangle^{\omega}\right)}{\partial t}}_{\text {Accumulation }}=\underbrace{\nabla \cdot\left[\varepsilon_{\omega} \mathbf{D}_{A \omega} \cdot\left(\nabla\left\langle c_{A \omega}\right\rangle^{\omega}+\frac{1}{V_{\omega}} \int_{A_{\omega \gamma}(t)} \mathbf{n}_{\omega \gamma} \tilde{c}_{A \omega} d A+\frac{1}{V_{\omega}} \int_{A_{\omega \kappa}(t)} \mathbf{n}_{\omega \kappa} \tilde{c}_{A \omega} d A\right)\right]}_{\text {Diffusion }} \\
& +\underbrace{\frac{1}{V} \int_{A_{\omega \gamma}(t)} \mathbf{n}_{\omega \gamma} \cdot\left(\mathbf{D}_{A \omega} \cdot \nabla c_{A \omega}\right) d A}_{\text {Interfacial Flux }} \\
& \underbrace{-\varepsilon_{\omega} \mu_{A \omega} \rho_{\omega} \frac{\left\langle c_{A \omega}\right\rangle^{\omega}}{\left\langle c_{A \omega}\right\rangle^{\omega}+K_{A \omega}} \frac{\left\langle c_{B \omega}\right\rangle^{\omega}}{\left\langle c_{B \omega}\right\rangle^{\omega}+K_{B \omega}}}_{\text {Reaction }}
\end{aligned}
$$

Averaged equation for the fluid ( $\gamma$-phase)

$$
\begin{aligned}
\underbrace{\frac{\partial\left(\varepsilon_{\gamma}\left\langle c_{A \gamma}\right\rangle^{\gamma}\right)}{\partial t}}_{\text {Accumulation }} & +\underbrace{\nabla \cdot\left(\varepsilon_{\gamma}\left\langle\mathbf{v}_{\gamma}\right\rangle^{\gamma}\left\langle c_{A \gamma}\right\rangle^{\gamma}\right)}_{\text {Convection }}= \\
& \underbrace{\nabla \cdot\left[\varepsilon_{\gamma} \mathcal{D}_{A \gamma}\left(\nabla\left\langle c_{A \gamma}\right\rangle^{\gamma}+\frac{1}{V_{\gamma}} \int_{A_{\omega \gamma}(t)} \mathbf{n}_{\gamma \omega} \tilde{c}_{A \gamma} d A+\frac{1}{V_{\gamma}} \int_{A_{\gamma K}(t)} \mathbf{n}_{\gamma K} \tilde{c}_{A \gamma} d A\right)\right]}_{\text {Diffusion }} \\
& +\underbrace{\frac{1}{V} \int_{A_{\omega \gamma}(t)} \mathbf{n}_{\gamma \omega} \cdot\left(\mathcal{D}_{A \gamma} \nabla c_{A \gamma}\right) d A}_{\text {Interfacial Flux }}-\underbrace{\nabla \cdot\left\langle\tilde{\mathbf{v}}_{\gamma} \tilde{c}_{A \gamma}\right\rangle}_{\begin{array}{c}
\text { Dispersive } \\
\text { Transport }
\end{array}}
\end{aligned}
$$

At this point, governing equations are unclosed in the sense that deviation quantities - which are 
unknown - still occur in integral terms. The next upscaling step consists in finding a way of system of balance equations in the case of local mass equilibrium can be found in [18]. In the present paper, we will focus on the derivation of the closed-forms of these macroscopic equations for two limit cases of non equilibrium.

\subsection{Two limit cases: reaction rate limited consumption (RRLC) and mass transfer limited consumption (MTLC)}

As emphasized above, different approaches are possible. First, a one-equation model can be classically derived from the unclosed averaged equations on the assumption of the existence of a local mass equilibrium between the $\omega$-phase and $\gamma$-phase. This condition implies a proportionality relationship between intrinsic averaged concentrations of both phases, i.e. $\left\langle c_{A \gamma}\right\rangle^{\gamma}=K_{A, e q}\left\langle c_{A \omega}\right\rangle^{\omega}$. As a consequence, averaged equations, Eqs. (15) and (16), can be summed up. Thus, interfacial flux terms are eliminated and one obtains a Darcy-scale description of solute transport in terms of a single averaged equation. This is the so-called Local Equilibrium Assumption model (LEA model). The limitation of this model is that the restrictive assumption of local mass equilibrium is valid only in the case of a diffusive regime of transport and at low reaction rate [18].

If characteristic times for transport are relatively different between the two regions or if the characteristic time for reaction is small compared with the characteristic time for transport in the $\omega$-phase, then non-equilibrium conditions exist. In principle, a two-medium description involving a separate mass balance equation for each phase could be adopted to adequately describe the mass transport within the system. Such a model is analogous to the "mobile-immobile" models classically used in solute transport for heterogeneous porous media exhibiting a non-Fickian behaviour (e.g. [1], [11]). In this context, convective and dispersive transport occurs in the more permeable zone, i.e., the fluid phase, called the "mobile" zone, while water inside the less 
permeable zone or "immobile" zone, i.e., the biofilm phase, is assumed to be stagnant. The mass exchange between the two regions is controlled by a diffusive process and is often macroscopically described by a first-order kinetics. A two-equation model is currently derived for application to bioremediation problems. However, the counterpart of this improved description is the increasing number of macroscopic effective coefficients that have to be determined and that depend on the properties of each phase in a complex way. Therefore, there is a real interest in developing a simplified Darcy-scale model of transport, whenever this is possible

Under certain conditions, indeed, non equilibrium one-equation models can also be derived from the non-closed averaged equations. In the case where the reaction is limited by mass transfer or kinetics, the interfacial flux term which appears in the macroscopic equation of the $\gamma$-phase may be obtained from a simplified formulation which is uncoupled from the equation of the $\omega$ phase. Calculation of the interfacial flux is then directly obtained from the solution of a closure problem. In Figure 3, we have illustrated the concentration profiles in the neighbourhood of the fluid-biofilm interface which result of the physical constraints associated with each considered model.

If the chemical reaction is limited by mass transfer between the fluid and biofilm phases, a MTLC (Mass Transfer Limited Consumption) model can be developed. Under this assumption, the concentration of substrate at the interface $A_{\gamma \omega}$ is considered as identically zero and we have:

$$
c_{A \gamma}=K_{A, e q} c_{A \omega}=0 \text {, at } A_{\gamma \omega}
$$

If the chemical reaction is limited by kinetics, which implies relatively low concentration gradients in the $\gamma$-phase, a RRLC (Reaction Rate Limited Consumption) model can be derived. This one-equation non-equilibrium model that has been widely adopted in chemical engineering uses the idea of an effectiveness factor to relate the (generally difficult to measure) reaction rate within the biofilm to the concentration in the bulk fluid-phase. In other words, we seek to discover conditions where it is meaningful to define a relationship for the rate of reaction of the form: 


$$
\varepsilon_{\omega} \mu_{A \omega} \rho_{\omega} \frac{\left\langle c_{A \omega}\right\rangle^{\omega}}{\left\langle c_{A \omega}\right\rangle^{\omega}+K_{A \omega}} \frac{\left\langle c_{B \omega}\right\rangle^{\omega}}{\left\langle c_{B \omega}\right\rangle^{\omega}+K_{B \omega}}=\eta \varepsilon_{\omega} \mu_{A \omega} \rho_{\omega} \frac{\left\langle c_{A \gamma}\right\rangle^{\gamma}}{\left\langle c_{A \gamma}\right\rangle^{\gamma}+K_{A \omega}} \frac{\left\langle c_{B \gamma}\right\rangle^{\gamma}}{\left\langle c_{B \gamma}\right\rangle^{\gamma}+K_{B \omega}}
$$

For the RRLC model, it is assumed that the deviation concentration $\tilde{c}_{A \gamma}$ is negligible relative to the intrinsic averaged concentration in the $\gamma$-phase, i.e.:

$$
\left\langle c_{A y}\right\rangle^{\gamma} \gg \tilde{c}_{A y}
$$

Under these conditions, the boundary equation (5) can be simplified to:

$$
K_{A, e q} c_{A \omega}=c_{A \gamma} \simeq\left\langle c_{A \gamma}\right\rangle^{\gamma}, \text { at } A_{\gamma \omega}
$$

Such approximations are usual in chemical engineering where they have been introduced heuristically (see for instance a general introduction about chemical reaction engineering in [11]).

\section{Development of the RRLC model}

If one assumes that the reaction rate is limited by the kinetics, i.e., the characteristic time of the biological reaction is very large compared to the characteristic time of the mass-transfer kinetics between phases, the boundary condition (5) can be simplified and replaced by:

$$
K_{A, e q} c_{A \omega}=c_{A \gamma}=\left\langle c_{A \gamma}\right\rangle^{\gamma} \text {, at } A_{\gamma \omega}
$$

The assumption here is that the concentration field in the $\omega$-phase depends only on the value of the intrinsic average concentration in the $\gamma$-phase through the boundary condition at the biofilmfluid interface. In the following we present the development of the averaged transport model based on this assumption.

\subsection{Closure}

To close the macroscopic transport equation, we must have a means of linking the deviation concentration $\tilde{c}_{A \gamma}$ to the intrinsic average concentration $\left\langle c_{A \gamma}\right\rangle^{\gamma}$. One can rearrange the definition of the deviation concentrations given in Eq. (14) to the form: 


$$
\tilde{c}_{A \gamma}=c_{A \gamma}-\left\langle c_{A \gamma}\right\rangle^{\gamma}
$$

This suggests that we can develop the governing differential equation for the deviation concentrations by subtracting the averaged equations from the point equations. To determine the mass balance equations for the deviation quantities, one can subtract Eq. (16) from Eq. (2). It can be shown (Appendix A) that the set of equations that predicts the deviation quantities for the RRLC model is given by:

$$
\begin{aligned}
& \mathbf{v}_{\gamma} \cdot \nabla \tilde{c}_{A \gamma}+\tilde{\mathbf{v}}_{\gamma} \cdot \nabla\left\langle c_{A \gamma}\right\rangle^{\gamma}=\nabla \cdot\left(\mathcal{D}_{A \gamma} \nabla \tilde{c}_{A \gamma}\right) \\
& -\frac{1}{V_{\gamma}} \int_{A_{\omega \gamma}(t)} \mathbf{n}_{\gamma \omega} \cdot\left(\mathcal{D}_{A \gamma} \nabla \tilde{c}_{A \gamma}\right) d A-\frac{1}{V_{\gamma}} \int_{A_{\gamma \kappa}(t)} \mathbf{n}_{\gamma \kappa} \cdot\left(\mathcal{D}_{A \gamma} \nabla \tilde{c}_{A \gamma}\right) d A \\
& \text { B.C.1 }-\mathbf{n}_{\gamma \kappa} \cdot \mathcal{D}_{A \gamma} \nabla \tilde{c}_{A \gamma}=\underbrace{\mathbf{n}_{\gamma \kappa} \cdot \mathcal{D}_{A \gamma} \nabla\left\langle c_{A \gamma}\right\rangle^{\gamma}}_{\text {source }} \text {, at } A_{\gamma \kappa}
\end{aligned}
$$$$
\text { B.C.2 } \tilde{c}_{A \gamma}=0, \text { at } \mathrm{A}_{\gamma \omega}
$$

Here, the non-homogeneous term $\nabla\left\langle c_{A \gamma}\right\rangle^{\gamma}$ acts as a 'source' term for the $\tilde{c}_{A \gamma}$ field in that it drives the equation. This problem is in fact equivalent to the active dispersion problem described by Quintard and Whitaker [38] in the case of local equilibrium in the flowing phase. Given the structure of the above problem, we may express the solution of these equations under the form:

$$
\tilde{c}_{A \gamma}=\mathbf{b}_{A \gamma} \cdot \nabla\left\langle c_{A \gamma}\right\rangle^{\gamma}
$$

where $\mathbf{b}_{A \gamma}$ is a vector field defined in the $\gamma$-phase. $\mathbf{b}_{A \gamma}$ is called a closure vector field or mapping vector field. By introducing this relation into deviation equations, given by Eqs. (23)-(25), and assuming the macroscopic source term spatially constant over the scale associated to the averaging volume, the set of equations governing the $\mathbf{b}_{A \gamma}$ field can be obtained. Note that in the averaged equations, Eqs. (15) and (16), the deviation concentration $\tilde{c}_{A \gamma}$ appears only in integral terms. De facto, the effective properties in these averaged equations are not particularly sensitive to the choice of boundary conditions provided that the averaging volume, $V$, is large enough so that it 
contains a large number of characteristic length scales $\left(\ell_{\omega}\right.$ and $\left.\ell_{\gamma}\right)$ [33]. Therefore, any local solution for $\tilde{c}_{A \gamma}$ that produces acceptable values of the area integrals can be used to determine the effective properties of the considered porous medium.

We will consider periodic conditions at the boundary of the unit cell because they usually impose less restrictive constraints on solutions than, for example, Dirichlet condition [18]. In addition, to solve the closure problem, it is necessary to introduce a condition that sets the level of $\mathbf{b}^{\prime}{ }_{A \gamma}$, and this condition is specified by a constraint of zero average per phase. In terms of the single periodic unit cell, the local closure problem can then be specified in the following dimensionless form.

\section{Closure problem I (dimensionless form)}

$$
\operatorname{Pe}\left(\tilde{\mathbf{v}}_{\gamma}^{\prime}+\mathbf{v}_{\gamma}^{\prime} \cdot \nabla \mathbf{b}_{A \gamma}^{\prime}\right)=\nabla^{2} \mathbf{b}^{\prime}{ }_{A \gamma}-\frac{1}{V_{\gamma}} \int_{A_{\omega \gamma}(t)} \mathbf{n}_{\gamma \omega} \cdot \nabla \mathbf{b}^{\prime}{ }_{A \gamma} d A-\frac{1}{V_{\gamma}} \int_{A_{\gamma \kappa}(t)} \mathbf{n}_{\gamma \kappa} \cdot \nabla \mathbf{b}_{A \gamma}{ }_{A \gamma} d A \text { in the } \gamma \text {-phase }
$$

B.C.1

$$
\begin{gathered}
-\mathbf{n}_{\gamma \kappa} \cdot \nabla \mathbf{b}_{A \gamma}^{\prime}=\mathbf{n}_{\gamma \kappa}, \quad \text { at } A_{\gamma \kappa} \\
\mathbf{b}_{A \gamma}^{\prime}=0, \quad \text { at } A_{\gamma \omega}(t)
\end{gathered}
$$

B.C.2

B.C.3

$$
\mathbf{b}^{\prime}{ }_{A \gamma}=\mathbf{b}^{\prime}{ }_{A \gamma}\left(\mathbf{r}+\mathbf{I}_{i}\right), \quad i=1,2,3, \quad \text { at } A_{\gamma e}
$$

B.C.4

$$
\left\langle\mathbf{b}^{\prime}{ }_{A \gamma}\right\rangle^{\gamma}=0
$$

where $A_{\gamma e}$ represents the areas of the entrances and exits of the $\gamma$-phases at the boundaries of the unit cell. In these expressions, the following dimensionless variables and parameters have been defined by:

$$
\begin{gathered}
\mathbf{b}_{A \gamma}^{\prime}=\frac{\mathbf{b}_{A \gamma}}{\ell_{\gamma}} \\
\tilde{\mathbf{v}}_{\gamma}^{\prime}=\frac{\tilde{\mathbf{v}}_{\gamma}}{\left\|\mathbf{v}_{\gamma}\right\|} \quad ; \quad \mathbf{v}_{\gamma}^{\prime}=\frac{\mathbf{v}_{\gamma}}{\left\|\mathbf{v}_{\gamma}\right\|}
\end{gathered}
$$


and we have used the notation:

$$
\left\|\mathbf{v}_{\gamma}\right\|=\left(\left\langle\mathbf{v}_{\gamma}\right\rangle^{\gamma} \cdot\left\langle\mathbf{v}_{\gamma}\right\rangle^{\gamma}\right)^{\frac{1}{2}}
$$

Using this closure solution, it follows that the averaged equation of transport takes the form:

Partially closed form of the averaged equation of transport

$$
\begin{array}{r}
\underbrace{\frac{\partial\left(\varepsilon_{\gamma}\left\langle c_{A \gamma}\right\rangle^{\gamma}\right)}{\partial t}}_{\text {Accumulation }}+\underbrace{\nabla \cdot\left(\varepsilon_{\gamma}\left\langle\mathbf{v}_{\gamma}\right\rangle^{\gamma}\left\langle c_{A \gamma}\right\rangle^{\gamma}\right)}_{\text {Convection }}=+\underbrace{\nabla \cdot\left(\mathbf{D}_{A, \text { eff }}^{*} \cdot \nabla\left\langle c_{A \gamma}\right\rangle^{\gamma}\right)}_{\text {Dispersion }} \\
+\underbrace{\frac{1}{V} \int_{A_{\omega \gamma}(t)} \mathbf{n}_{\gamma \omega} \cdot\left(\mathcal{D}_{A \gamma} \nabla c_{A \gamma}\right) d A+\frac{1}{V} \int_{A_{\omega \kappa}(t)} \mathbf{n}_{\gamma \kappa} \cdot\left(\mathcal{D}_{A \gamma} \nabla c_{A \gamma}\right) d A}_{\text {Interfacial Flux }}
\end{array}
$$

where the effective dispersion tensor for the species $A, \mathbf{D}_{A, \text { eff }}^{*}$, is given by:

$$
\mathbf{D}_{A, e f f}^{*}=\varepsilon_{\gamma} \mathcal{D}_{A \gamma}\left(\mathbf{I}+\frac{1}{V_{\gamma}} \int_{A_{\omega \gamma}(t)} \mathbf{n}_{\gamma \omega} \mathbf{b}_{A \gamma} d A+\frac{1}{V_{\gamma}} \int_{A_{\gamma K}(t)} \mathbf{n}_{\gamma \kappa} \mathbf{b}_{A \gamma} d A\right)-\left\langle\tilde{\mathbf{v}}_{\gamma} \mathbf{b}_{A \gamma}\right\rangle
$$

However, this problem is not yet fully closed because the microscale concentrations still appear in the interfacial flux terms. In order to obtain a fully closed averaged equation of transport, we just need to find an expression of the interfacial flux as a function of the macroscopic quantities.

\subsection{Derivation of the interfacial flux (Appendix B)}

Let us consider the diffusive fluxes of substrate through the boundary of the $\gamma$-phase defined in Eq. (16). Given the boundary condition stated by Eq. (4), we can rewrite the interfacial flux in the following form:

$$
\frac{1}{V} \int_{A_{\omega \gamma}(t)} \mathbf{n}_{\gamma \omega} \cdot\left(\mathcal{D}_{A \gamma} \nabla c_{A \gamma}\right) d A+\frac{1}{V} \int_{A_{\omega \kappa}(t)} \mathbf{n}_{\gamma \kappa} \cdot\left(\mathcal{D}_{A \gamma} \nabla c_{A \gamma}\right) d A=\frac{1}{V} \int_{A_{\omega \gamma}(t)} \mathbf{n}_{\gamma \omega} \cdot\left(\mathcal{D}_{A \gamma} \nabla c_{A \gamma}\right) d A
$$

Then, on the basis of the boundary condition given by Eq. (6), this result can be expressed as: 


$$
\frac{1}{V} \int_{A_{\omega \gamma}(t)} \mathbf{n}_{\gamma \omega} \cdot\left(\mathcal{D}_{A \gamma} \nabla c_{A \gamma}\right) d A=\frac{1}{V} \int_{A_{\omega \gamma}(t)} \mathbf{n}_{\gamma \omega} \cdot\left(\mathbf{D}_{A \omega} \cdot \nabla c_{A \omega}\right) d A
$$

As the reaction kinetics is assumed to be slow enough relative to external mass transfer, it means that spatial concentration gradients are negligible in the fluid phase. Thus, pore-scale equations can be uncoupled and the initial boundary value problem for $c_{A \omega}$ is put under the form:

$\gamma$-phase transport equation

$$
c_{A \gamma}=\left\langle c_{A \gamma}\right\rangle^{\gamma} \quad \text { in the } \gamma \text {-phase }
$$

$\omega$-phase transport equation

$$
\frac{\partial c_{A \omega}}{\partial t}=\nabla \cdot\left(\mathbf{D}_{A \omega} \cdot \nabla c_{A \omega}\right)-\mu_{A \omega} \rho_{\omega} \frac{c_{A \omega}}{c_{A \omega}+K_{A \omega}} \frac{c_{B \omega}}{c_{B \omega}+K_{B \omega}} \quad \text { in the } \omega \text {-phase, }
$$

B.C.1

$$
c_{A \omega}=K_{A, e q}^{-1}\left\langle c_{A \gamma}\right\rangle^{\gamma}, \text { at } A_{\gamma \omega}(t)
$$

B.C.2

$$
-\mathbf{n}_{\omega \kappa} \cdot \mathbf{D}_{A \omega} \cdot \nabla c_{A \omega}=0 \text { at } A_{\omega \kappa}(t)
$$

At this point, we identify $\left\langle c_{A \gamma}\right\rangle^{\gamma}$ as the source term of the microscopic field $c_{A \omega}$. In other words, we directly express the concentration $c_{A \omega}$ as a function of the intrinsic averaged concentration in the $\gamma$-phase and a scalar field $S$ defined in the $\omega$-phase, i.e.:

$$
c_{A \omega}=K_{A, e q}^{-1} S\left\langle c_{A \gamma}\right\rangle^{\gamma}
$$

We can then rewrite the interfacial flux as follows:

$$
\frac{1}{V} \int_{A_{\omega \gamma}(t)} \mathbf{n}_{\gamma \omega} \cdot\left(\mathcal{D}_{A \gamma} \nabla c_{A \gamma}\right) d A=\frac{K_{A, e q}{ }^{-1}\left\langle c_{A \gamma}\right\rangle^{\gamma}}{V} \int_{A_{\omega \gamma}(t)} \mathbf{n}_{\gamma \omega} \cdot\left(\mathbf{D}_{A \omega} \cdot \nabla S\right) d A
$$

which finally leads to (see Appendix B):

$$
\frac{1}{V} \int_{A_{\omega \gamma}(t)} \mathbf{n}_{\gamma \omega} \cdot\left(\mathcal{D}_{A \gamma} \nabla c_{A \gamma}\right) d A=\eta \varepsilon_{\omega} \mu_{A \omega} \rho_{\omega} \frac{K_{A, e q}^{-1}\left\langle c_{A \gamma}\right\rangle^{\gamma}}{K_{A, e q}^{-1}\left\langle c_{A \gamma}\right\rangle^{\gamma}+K_{A \omega}} \frac{\left\langle c_{B \omega}\right\rangle^{\omega}}{\left\langle c_{B \omega}\right\rangle^{\omega}+K_{B \omega}}
$$

with an effectiveness factor $\eta$ defined as follows: 


$$
\eta=\frac{\left(K_{e f f}+1\right)}{c_{B \omega}^{*} \varepsilon_{\omega} D a K_{e f f}} \int_{A_{f o}} \mathbf{n}_{\gamma \omega} \cdot \nabla S d A
$$

The effectiveness factor, classically used in chemical engineering [11], takes a value between 0 and 1 and represents physically the observed reaction rate versus the maximal reaction rate, i.e., the reaction that would occur if the entire volume is exposed to the bulk concentration. In this expression, the following dimensionless variables and parameters have been defined by:

$$
\begin{gathered}
D a=\frac{\ell_{\omega}^{2} \mu_{A \omega} \rho_{\omega}}{K_{A \omega} \mathcal{D}_{A \omega}} \\
K_{e f f}=\frac{K_{A \omega}}{\left\langle c_{A \gamma}\right\rangle^{\gamma} K_{A, e q}^{-1}} \\
c_{B \omega}^{*}=\frac{\left\langle c_{B \omega}\right\rangle^{\omega}}{\left\langle c_{B \omega}\right\rangle^{\omega}+K_{B \omega}}
\end{gathered}
$$

Here, $\mathcal{D}_{A \omega}$ is the current component of the diffusion tensor in the $\omega$-phase, supposed to be spherical for simplicity. $D a$ is the Damköhler number associated with the reaction of biodegradation of substrate $A$. Classically, the effectiveness factor is expressed in the literature [6] as a function of the Damköhler number which is the ratio between a characteristic time scale of diffusion for the considered substrate and a characteristic time scale of reaction. Note that the reference length of these dimensionless numbers, $\ell_{\omega}$, is different from the one used in the expression of the Péclet number, equations (32) and (34), denoted $\ell_{\gamma} \cdot \ell_{\omega}$ is the characteristic length of the $\omega$-phase (associated to the reactive region) at the microscopic scale, while $\ell_{\gamma}$ is the characteristic length of the $\gamma$-phase (associated to the convective region) at the microscopic scale. Since the reaction has hyperbolic kinetics, an additional dimensionless number appears in Eq. (47) . The second dimensionless characteristic number is $K_{e f f}$, the effective half saturation constant of the reaction. If $K_{\text {eff }}$ tends towards infinity (for a very low macroscopic concentration for 
example), the non-linear kinetics of reaction follows first order kinetics, and if $K_{\text {eff }}$ is close to zero (e.g., in the case of a very high macroscopic concentration), the reaction tends to zero order kinetics. One must note that $K_{\text {eff }}$ depends on the macroscopic variable $\left\langle c_{A \gamma}\right\rangle^{\gamma}$; this introduces a coupling between the pore-scale calculation of $\eta$ and the solution of the macroscopic transport equation.

In order to calculate the scalar field $S$, we substitute Eq. (44) in Eq. (1). At this point, we use the classical quasi-steady assumption which means the characteristic relaxation time of the microscopic quantity $c_{A \omega}$ is considered to be very small compared to the characteristic time of variation of its source term, the macroscopic quantity $\left\langle c_{A \gamma}\right\rangle^{\gamma}$. This separation of time scales is directly related to the constraint of separation of space scales previously detailed - see [36] for more details. Under this assumption, we obtain the following dimensionless problem which determines the scalar field $S$ :

\section{Closure problem II}

$$
\begin{aligned}
& \text { B.C.1 } \mathbf{n}_{\omega \kappa} \cdot \nabla S=0 \text { at } \mathrm{A}_{\omega \kappa}(t) \\
& \text { B.C.2 } \quad S=1 \text { at } \mathrm{A}_{\gamma \omega}(t)
\end{aligned}
$$

By solving this problem on the $\omega$-phase of the unit cell which defines the microscale structure of the medium (used also for the calculation of the closure vector field $\mathbf{b}_{A \gamma}$, see part 4.1.), one can evaluate the effectiveness factor $\eta$, and hence the interfacial flux. Note that in Eqs. (51) and (47) also appears the term $c_{B \omega}^{*}$. Consequently, in the case of a two-species transport problem, in addition to the coupling through $K_{\text {eff }}$ with the macroscopic concentration $\left\langle c_{A \gamma}\right\rangle^{\gamma}$ mentioned 
above, the calculation of the effectiveness factor for species A is also dependent on the solving of

From the mathematical formalism point of view, it is interesting to note that the approach followed here is in the spirit of the work of Rao et al [40] for solute transport in bimodal porous medium. They have developed a fully transient solution for the mass transfer coefficient between two regions (equivalent, in our reactive case, to the effectiveness factor) under the following simplified conditions: the transport within the inclusions is only driven by diffusion, spatial concentration gradients are negligible in the fluid phase and the inclusions are non-interacting uniform spheres (or cylinders). Under such assumptions, the resulting closure problem is similar in its formulation to the one obtained for the calculation of the effectiveness factor. In essence, these two approaches, Rao's one and ours, can be regarded as belonging to the socalled class of mixed models ([5], [17]). Basically, for a two-phase system, this kind of description is based on a fully averaged equation associated to the connected medium, coupled to a lowerscale model (associated to the biofilm phase or inclusion region). Note that the expression of the dispersion tensor, given by Eq. (37), is also typical of mixed models: see for instance Eqs. (57)(62) of the closure problem in [17]. A typical example of such a model is presented in this previous work, which should be consulted for more details.

\subsection{Closed form of the RRLC macroscopic model}

Finally, the resulting closed form of the RRLC macroscopic model can be written as follows:

$$
\begin{aligned}
\underbrace{\frac{\partial\left(\varepsilon_{\gamma}\left\langle c_{A \gamma}\right\rangle^{\gamma}\right)}{\partial t}}_{\text {Accumulation }} & +\underbrace{\nabla \cdot\left(\varepsilon_{\gamma}\left\langle\mathbf{v}_{\gamma}\right\rangle^{\gamma}\left\langle c_{A \gamma}\right\rangle^{\gamma}\right)}_{\text {Convection }}=\underbrace{\nabla \cdot\left(\mathbf{D}_{A, e f f}^{*} \cdot \nabla\left\langle c_{A \gamma}\right\rangle^{\gamma}\right)}_{\text {Dispersion }} \\
& -\underbrace{\eta \varepsilon_{\omega} \mu_{A \omega} \rho_{\omega} \frac{K_{A, e q}^{-1}\left\langle c_{A \gamma}\right\rangle^{\gamma}}{K_{A, e q}^{-1}\left\langle c_{A \nu}\right\rangle^{\gamma}+K_{A \omega}} \frac{\left\langle c_{B \gamma}\right\rangle^{\gamma}}{\left\langle c_{B \gamma}\right\rangle^{\gamma}+K_{B \omega}}}_{\text {Interfacial Flux }}
\end{aligned}
$$

where the effective dispersion tensor for the species $A, \mathbf{D}_{A, \text { eff }}^{*}$, is given by: 


$$
\mathbf{D}_{A, e f f}^{*}=\varepsilon_{\gamma} \mathcal{D}_{A \gamma} \mathbf{I}+\mathcal{D}_{A \gamma}\left(\frac{1}{V} \int_{A_{\omega \gamma}(t)} \mathbf{n}_{\gamma\left(\mathbf{b}_{A \gamma}\right.} d A+\frac{1}{V} \int_{A_{\mu k}(t)} \mathbf{n}_{\gamma \kappa} \mathbf{b}_{A \gamma} d A\right)-\left\langle\tilde{\mathbf{v}}_{\gamma} \mathbf{b}_{A \gamma}\right\rangle
$$

and the reaction effectiveness factor $\eta$ takes the form:

$$
\eta=\frac{\left(K_{e f f}+1\right)}{c_{B \omega}^{*} \varepsilon_{\omega} K_{e f f} D a} \int_{A_{\gamma \omega}} \mathbf{n}_{\gamma \omega} \cdot \nabla S d A
$$

\section{Development of the MTLC model}

On the contrary, if one assumes that the reaction rate is limited by the external mass transfer, i.e., the mass-transfer kinetics through $A_{\gamma \omega}$ is very slow compared to the reaction kinetics, the solute is instantaneously consumed as soon as it penetrates inside the biofilm phase. As a consequence, the substrate concentration is identically zero within the $\omega$-phase, i.e.:

$$
c_{A \omega}=0 \text { in } \omega \text {-phase }
$$

and the boundary condition at the fluid-biofilm interface, Eq. (5), becomes:

$$
c_{A \gamma}=K_{A, e q} c_{A \omega}=0, \text { at } A_{\gamma \omega}
$$

It must be emphasized here that there is a parallel between the microscale problem developed above and the set of equations associated to any reaction systems exhibiting mass transfer limitations; we can cite for instance the problem of dissolution in porous media with a mass transfer limited kinetics studied by Quintard and Whitaker [38] and Golfier et al. [15]. In both cases, the assumption on limiting mechanisms leads to the same formalism at the pore-scale. Therefore, the development of the averaged transport equation is similar to the one presented in these papers. Nevertheless, as the derivation of such a model is not straightforward, we shall remind the different steps and assumptions made so that the limitations and problems associated to 
this analysis appear clearly. Additional details can be found in these previous papers ([38], [15]).

\subsection{Derivation of the closure problem}

If the development of the deviation equations (23) and (24) of the RRLC model (Appendix A) is similar in the case of the MTLC model, the expression of the second boundary condition, given by equation (25), is quite different. At this point, the boundary value problem for the deviation concentration $\tilde{c}_{A \gamma}$ in the case of the MTLC model takes the form:

$$
\begin{aligned}
\frac{\partial \tilde{c}_{A \gamma}}{\partial t}+\tilde{\mathbf{v}}_{\gamma} \cdot \nabla\left\langle c_{A \gamma}\right\rangle^{\gamma}+\mathbf{v}_{\gamma} \cdot \nabla \tilde{c}_{A \gamma} & =\nabla \cdot\left(\mathcal{D}_{A \gamma} \nabla \tilde{c}_{A \gamma}\right) \\
& -\nabla \cdot\left[\mathbf{D}_{A \gamma}\left(\frac{1}{V_{\gamma}} \int_{A_{\omega \gamma}(t)} \mathbf{n}_{\gamma \omega} \tilde{c}_{A \gamma} d A+\frac{1}{V_{\gamma}} \int_{A_{\omega \kappa}(t)} \mathbf{n}_{\gamma \kappa} \tilde{c}_{A \gamma} d A\right)\right]
\end{aligned}
$$

B.C.1

$$
-\mathbf{n}_{\gamma \kappa} \cdot \mathcal{D}_{A \gamma} \nabla \tilde{c}_{A \gamma}=\underbrace{\mathbf{n}_{\gamma \kappa} \cdot \mathcal{D}_{A \gamma} \nabla\left\langle c_{A \gamma}\right\rangle^{\gamma}}_{\text {source }}, \quad \text { at } A_{\gamma \kappa}
$$

B.C.2

$$
\tilde{c}_{A \gamma}=\underbrace{-\left\langle c_{A \gamma}\right\rangle^{\gamma}}_{\text {source }}, \text { at } \mathrm{A}_{\gamma \omega}
$$

One can identify $\nabla\left\langle c_{A \gamma}\right\rangle^{\gamma}$ and $\left\langle c_{A \gamma}\right\rangle^{\gamma}$ as source terms of the $\tilde{c}_{A \gamma}$ field. This leads to express the solution to these equations in the form (see [14]):

$$
\tilde{c}_{A \gamma}=\mathbf{b}_{A \gamma} \cdot \nabla\left\langle c_{A \gamma}\right\rangle^{\gamma}-s_{A \gamma}\left\langle c_{A \gamma}\right\rangle^{\gamma}
$$

where $\mathbf{b}_{A \gamma}$ is a vector closure field and $s_{A \gamma}$ is a scalar closure field. If we inject this expression in equations (59) to (61), we obtain the two following closure problems, both defined in the $\gamma$-phase:

\section{Closure problem I}

$$
\operatorname{Pe}\left(\tilde{\mathbf{v}}_{\gamma}+\mathbf{v}_{\gamma}^{\prime} \cdot \nabla \mathbf{b}_{A \gamma}^{\prime}\right)=\nabla^{2} \mathbf{b}_{A \gamma}^{\prime}-\frac{1}{V_{\gamma}} \int_{A_{\omega \gamma}} \mathbf{n}_{\gamma \omega} \cdot \nabla \mathbf{b}_{A \gamma}^{\prime} d A-\frac{1}{V_{\gamma}} \int_{A_{\kappa \gamma}} \mathbf{n}_{\gamma \kappa} \cdot \nabla \mathbf{b}_{A \gamma}^{\prime} d A \quad \text { in the } \gamma \text {-phase }
$$

B.C.1

$$
-\mathbf{n}_{\gamma \kappa}=\mathbf{n}_{\gamma \kappa} \cdot \nabla \mathbf{b}_{A \gamma}^{\prime} \text { at } A_{\gamma \kappa}
$$

B.C. 2

$$
\mathbf{b}_{A \gamma}^{\prime}=0 \text { at } A_{\gamma \omega}
$$


B.C.3

$\mathbf{b}_{A \gamma}^{\prime}=\mathbf{b}_{A \gamma}{ }_{A \gamma}\left(\mathbf{r}+\mathbf{I}_{i}\right), \quad i=1,2,3, \quad$ at $A_{\gamma e}$

B.C. 4

$\left\langle\mathbf{b}^{\prime}{ }_{A \gamma}\right\rangle^{\gamma}=0$

Closure problem II

B.C.1

$$
P e \mathbf{v}_{\gamma}^{\prime} \cdot \nabla s_{A \gamma}=\nabla^{2} s_{A \gamma}-\frac{1}{V_{\gamma}} \int_{A_{\omega \gamma}} \mathbf{n}_{\gamma \omega} \cdot \nabla s_{A \gamma} d A \text { in the } \gamma \text {-phase }
$$

B.C.2

$$
s_{A \gamma}=1 \text { at } A_{\gamma \omega}
$$

B.C.3

$$
s_{A \gamma}=s_{A \gamma}\left(\mathbf{r}+\mathbf{I}_{i}\right), \quad i=1,2,3, \quad \text { at } A_{\gamma e}
$$

B.C. 4

$$
\left\langle s_{A \gamma}\right\rangle^{\gamma}=0
$$

In these expressions, dimensionless variables and parameters have been defined as previously in Eqs. (32)-(35). At first sight, the closure problem I of the MTLC and RRLC models are identical. Actually, it must be emphasized that such a similarity is not inherent to these models but due to certain upscaling assumptions. Indeed, it is classically assumed that the space variations of macroscale variables $\left(\left\langle c_{A \gamma}\right\rangle^{\gamma}\right.$ and $\left.\nabla\left\langle c_{A \gamma}\right\rangle^{\gamma}\right)$ at the pore scale are neglected in the development of closure problems [55]. However, for certain specific configurations such as the tube problem for instance, several studies ([25], [16]) have shown that keeping these terms could lead to an improvement of the solution. In this case, the closure problem I of the MTLC model would be different and coupled with Problem II. Given the kind of application under consideration and for the sake of simplicity, we will keep the classical approach.

\subsection{Closed form of the MTLC macroscopic model}

By injecting Eq. (62) into Eq. (16), we finally obtain the macroscopic equation of transport associated with the MTLC model: 


$$
\begin{aligned}
& \frac{\partial\left(\varepsilon_{\gamma}\left\langle c_{A \gamma}\right\rangle^{\gamma}\right)}{\partial t}+\nabla \cdot\left(\varepsilon_{\gamma}\left\langle\mathbf{v}_{\gamma}\right\rangle^{\gamma}\left\langle c_{A \gamma}\right\rangle^{\gamma}\right)= \\
& \nabla \cdot\left(\mathbf{D}_{A, e f f}^{*} \cdot \nabla\left\langle c_{A \gamma}\right\rangle^{\gamma}\right)-\alpha\left\langle c_{A \gamma}\right\rangle^{\gamma}+\nabla \cdot\left(\mathbf{d}_{\gamma}^{*}\left\langle c_{A \gamma}\right\rangle^{\gamma}\right)+\left(\mathbf{u}_{\gamma}^{*}-\mathcal{D}_{A \gamma} \nabla \varepsilon_{\gamma}\right) \cdot \nabla\left\langle c_{A \gamma}\right\rangle^{\gamma}
\end{aligned}
$$

where the different macroscopic parameters are defined by:

$$
\begin{gathered}
\mathbf{D}_{A, e f f}^{*}=\varepsilon_{\gamma} \mathcal{D}_{A \gamma}+\mathcal{D}_{A \gamma}\left(\frac{1}{V} \int_{A_{\omega \gamma}} \mathbf{n}_{\gamma \sigma} \mathbf{b}_{A \gamma} d A+\frac{1}{V} \int_{A_{\alpha \gamma}} \mathbf{n}_{\gamma K} \mathbf{b}_{A \gamma} d A\right)-\left\langle\tilde{\mathbf{v}}_{\gamma} \mathbf{b}_{A \gamma}\right\rangle \\
\alpha=\frac{1}{V} \int_{A_{o \gamma}} \mathbf{n}_{\gamma \omega} \cdot \mathcal{D}_{A \gamma} \nabla s_{A \gamma} d A \\
\mathbf{d}_{\gamma}^{*}=-\mathcal{D}_{A \gamma}\left(\frac{1}{V} \int_{A_{\omega \gamma}} \mathbf{n}_{\gamma \omega} s_{A \gamma} d A+\frac{1}{V} \int_{A_{\alpha \gamma}} \mathbf{n}_{\gamma \kappa} s_{A \gamma} d A\right)+\left\langle\tilde{\mathbf{v}}_{\gamma} s_{A \gamma}\right\rangle \\
\mathbf{u}_{\gamma}^{*}=\frac{1}{V} \int_{A_{\omega \gamma}} \mathbf{n}_{\gamma \omega} \cdot \mathcal{D}_{A \gamma} \nabla \mathbf{b}_{A \gamma} d A+\frac{1}{V} \int_{A_{k \gamma}} \mathbf{n}_{\gamma \kappa} \cdot \mathcal{D}_{A \gamma} \nabla \mathbf{b}_{A \gamma} d A
\end{gathered}
$$

Here, $\mathbf{D}_{A, \text { eff }}^{*}$ is the macroscopic dispersion tensor, $\alpha$ is the exchange coefficient between the $\gamma$ phase and the $\omega$-phase and $\mathbf{d}_{\gamma}^{*}$ and $\mathbf{u}_{\gamma}^{*}$ are non-classical convective vector parameters. As emphasized previously, the effective dispersion calculated with the MTLC model (closure problem I) is equivalent to the dispersion tensor predicted by the RRLC model. It is quite surprising that both of these opposing cases yield the same assumptions regarding the dispersion process (subject to the upscaling conditions stated above). One must also keep in mind that the mass exchange coefficient $\alpha$ introduced above corresponds to a first order approximation of the mass transfer processes within the system, which, by nature, are transient and require a spatialtime convolution to be rigorously described. The limitations associated to this approximation and the conditions for which such a formulation may be not acceptable are discussed in Cherblanc et al. [8] and Golfier et al. [17]. Finally, it was shown that the additional terms $\nabla \cdot\left(\mathbf{d}_{\gamma}^{*}\left\langle c_{A \gamma}\right\rangle^{\gamma}\right)$ and $\left(\mathbf{u}_{\gamma}^{*}-\mathcal{D}_{A \gamma} \nabla \varepsilon_{\gamma}\right) \cdot \nabla\left\langle c_{A \gamma}\right\rangle^{\gamma}$ have a complex influence on the solution behavior. In general, for simple unit cells, these additional terms are necessary. On the contrary, for more complex microscale 
geometries, results are contradictory and we currently lack information ([39], [15], [16]). The

\section{Numerical implementation and validation of the non equilibrium models}

A key point in the derivation of upscaled models devised above is the calculation of the interfacial flux. Indeed, in both formulations, the solute transport equation is averaged over the $\gamma$-phase and the mass exchange flux with the biofilm is governed only by the value of the effectiveness factor or the mass transfer coefficient. In this section, we will study the behaviour and accuracy of these interfacial fluxes through two simple examples, by comparing numerical results with analytical or semi analytical solutions. In a first step, numerical methods used for solving closure problems will be briefly presented. Finally, the domains of validity of each model will be assessed by comparison between direct simulations at the pore scale over a stratified porous medium and results coming from 1D averaged models.

\subsection{Numerical methods}

The numerical procedure designed for solving the closure problems has been described in the literature (e.g., [38]). Effective coefficients in transport equations are computed for a given 
geometry and a velocity field previously calculated. An example of the simple representation of the 'solid/biofilm' microstructure that was adopted is represented by the two-dimensional periodic unit cell illustrated in Figure 4. The porous medium represented here is constituted by a cylindrical solid inclusion, with a biofilm layer, uniformly developed around the inclusion. On this figure, $\ell_{\gamma}$ and $\ell_{\omega}$ represent the characteristic length scales associated to the fluid and biofilm phase, respectively. The equations are discretized on a two-dimensional uniform Cartesian grid. The geometry of the medium under consideration is described by assigning fluid, biofilm or solid properties to each block of the Cartesian grid. A grid of 250x250 nodes is used and provides sufficient resolution to obtain accurate results.

These problems are numerically solved with a finite volume scheme. The velocity field is initially computed over the given unit cell. The discretization of the Stokes equations does not pose major problems and a classical Uzawa algorithm [13] is considered to update the pressure until convergence. The convective part of the closure equations, when it exists, has been discretized with a first order upstream scheme with anti-diffusion [41] and the dispersive part has been discretized with an implicit scheme [37]. The difficulties generated by the integrodifferential terms and the conditions of zero average in the different phases of the solution field have been overcome by a method of decomposition of variables (e.g. [38]). The resulting linear systems have been solved by a Bi-Conjugate Gradient Stabilized solver [49]. Unless otherwise indicated, the results for the different effective parameters are represented in terms of the cell Péclet number (see Eqs. (34) and (35).

\subsection{Interfacial flux predicted by the RRLC model: application to a one-dimensional stratified medium}

We present here a validation of the effectiveness factor calculation obtained from the upscaling theory; this is based on a comparison with an analytical solution in the case of a one-dimensional 
stratified porous medium. Figure 5 illustrates such a conceptual model, commonly adopted in the bulk fluid phase, assumed to be perfectly mixed and a biofilm region where confined concentration gradients may exist due to the diffusion and reaction processes. No boundary layer, including a diffusion resistance, as in the film stagnant or boundary layer theory, is considered here (e.g. [4], [24]).

In order to be able to reach an analytical solution, we will consider linear first order kinetics for the reaction of substrate consumption in the biofilm. Under the assumption of reaction rate limited kinetics, the set of microscopic equations describing the solute transport through the porous medium (Eqs. (1) to (6)) can thus be written as:

$\gamma$-phase

$$
c_{A \gamma}=\left\langle c_{A \gamma}\right\rangle^{\gamma} \quad \text { in the } \gamma \text {-phase }
$$

$\omega$-phase

$$
\frac{\partial c_{A \omega}}{\partial t}=\mathcal{D}_{A \omega} \frac{\partial^{2} c_{A \omega}}{\partial x^{2}}-k_{A \omega} c_{A \omega} \quad \text { in the } \omega \text {-phase }
$$

B.C. 1

$$
-\mathcal{D}_{A \omega} \frac{\partial c_{A \omega}}{\partial x}=0 \quad \text { at } X_{\omega \kappa}
$$

\section{B.C. 2}

$$
c_{A \omega}=c_{A \gamma}=\left\langle c_{A \gamma}\right\rangle^{\gamma}, \text { at } X_{\omega \gamma}
$$

where $k_{A \omega}$ is the first-order reaction rate of solute biodegradation. A straightforward calculation leads to the analytical expression of the effectiveness factor in this case:

$$
\eta_{\text {analytic }}=\frac{1}{\sqrt{D a_{1}}\left(X_{\omega \kappa}-X_{\omega \gamma}\right)} \frac{\sinh \left(\sqrt{D a_{1}} X_{\omega \kappa}\left(1-\frac{X_{\omega \gamma}}{X_{\omega \kappa}}\right)\right)}{\cosh \left(\sqrt{D a_{1}} X_{\omega \kappa}\left(1-\frac{X_{\omega \gamma}}{X_{\omega \kappa}}\right)\right)}
$$


with a Damköhler number $D a_{1}$ defined by:

$$
D a_{1}=\frac{k_{A \omega} L^{2}}{\mathcal{D}_{A \omega}}
$$

where the characteristic length $L$ is here equal to 1 . This analytical result, which is classical in chemical engineering, is compared to the effectiveness factor value given by the RRLC model (modified to consider a first order linear reaction kinetics).

The variation of $\eta$ as a function of the Damköhler number is illustrated in Figure 6. An excellent agreement between analytical and numerical results is obtained, with an averaged absolute relative error of less than $1 \%$.

\subsection{Interfacial flux predicted by the MTLC model: application to a capillary tube model}

At this time, it is well known that a constant mass transfer coefficient like the one calculated from the volume averaging method cannot recover all the complex physical processes involved, which are inherently both transient and spatially dependent ([17], [25]). For instance, the derivation of the closure problem that controls $\tilde{c}_{A \gamma}$ implies that macroscopic gradients are assumed to be small compared to microscopic values. Thus, the effects associated to the entrance region are not considered. For a better understanding of the influence of such effects on the MTLC model, let us consider a simplified porous medium such as the capillary tube model in which the pore space is represented as an array of parallel cylindrical tubes. The biofilm is assumed to be developed as a uniform layer on the walls of each tube. Considering the axisymmetry of the problem, the velocity field is such as $\mathbf{v}_{\gamma}=v_{\gamma}(r) . \mathbf{e}_{\mathbf{z}}$, with $r$ the radial coordinates and $\mathbf{e}_{\mathbf{z}}$ the unit vector on the axial direction. The representative pore scale geometry of such a conceptual system is illustrated in Figure 7. The flow and boundary conditions used to calculate 
the mass transfer coefficient $\alpha$ in the framework of the MTLC model are also indicated on this Figure.

First, let us remind that the mass transfer coefficient $\alpha$ can be put in a dimensionless form, which leads to a Sherwood number $S h$ defined as follows:

$$
S h=\frac{\alpha D}{a_{v} \mathcal{D}_{A \gamma}}
$$

where $D$ is the diameter of the tube and $a_{v}$ is the specific surface of the medium (i.e., the total exchange surface area per unit of volume). In this axi-symmetric configuration, this Sherwood number can be rewritten under the form:

$$
S h=\frac{\alpha R^{2}}{\mathcal{D}_{A \gamma}}
$$

where $R$ is the internal tube radius. Actually, this conceptual model is analogous to the classical Graetz problem for heat (or mass by analogy) transport [23] driven by the following macroscopic balance equation,

$$
\frac{\partial\left\langle c_{A \gamma}\right\rangle^{\gamma}}{\partial t}+\left\langle\mathbf{v}_{\gamma}\right\rangle^{\gamma} \cdot \nabla\left\langle c_{A \gamma}\right\rangle^{\gamma}=\mathcal{D}_{\text {Taylor }}^{*} \nabla^{2}\left\langle c_{A \gamma}\right\rangle^{\gamma}-\alpha_{\text {Graetz }}\left\langle c_{A \gamma}\right\rangle^{\gamma}
$$

for which a semi-analytical transient solution is available. Here, $\mathcal{D}_{\text {Taylor }}^{*}$ represents the axial dispersion coefficient characteristic of Taylor dispersion [48], $\alpha_{\text {Graetz }}$ being the mass transfer coefficient predicted by the Graetz analysis. Initially, the fully transient expression obtained by Graetz was derived for the bulk average concentration but a similar calculation can be made as a function of $\left\langle c_{A \gamma}\right\rangle^{\gamma}$.

In order to compare both expressions of the mass transfer coefficient, the formulation of the MTLC model needs to be modified. Thus, the macroscale transport equation becomes:

$$
\frac{\partial\left(\left\langle c_{A \gamma}\right\rangle^{\gamma}\right)}{\partial t}+\nabla \cdot\left(\mathbf{v}_{e f f}\left\langle c_{A \gamma}\right\rangle^{\gamma}\right)=\nabla \cdot\left(\varepsilon_{\gamma}^{-1} \mathbf{D}_{A, e f f}^{*} \cdot \nabla\left\langle c_{A \gamma}\right\rangle^{\gamma}\right)-\alpha^{\prime}\left\langle c_{A \gamma}\right\rangle^{\gamma}
$$


with:

$$
\alpha^{\prime}=\varepsilon_{\gamma}^{-1} \alpha
$$

and:

$$
\mathbf{v}_{e f f}=\left\langle\mathbf{v}_{\gamma}\right\rangle^{\gamma}-\varepsilon_{\gamma}^{-1} \mathbf{d}_{\gamma}^{*}-\varepsilon_{\gamma}^{-1} \mathbf{u}_{\gamma}^{*}
$$

where $\mathbf{v}_{\text {eff }}$ is the effective velocity associated with the convection term. As we can notice from Eqs. (87) and (88), advected mass fluxes are not the same. However, by considering a steadystate assumption and negligible axial dispersion, the coefficient $\alpha^{\prime}$ can be redefined in a similar form to $\alpha_{\text {Graetz }}$, as a function of the classical averaged fluid velocity $\left\langle\mathbf{v}_{\gamma}\right\rangle^{\gamma}$, such as:

$$
\alpha^{*}=\frac{\alpha^{\prime}\left\|\left\langle\mathbf{v}_{\gamma}\right\rangle^{\gamma}\right\|}{\left\|\left(\left\langle\mathbf{v}_{\gamma}\right\rangle^{\gamma}-\varepsilon_{\gamma}^{-1} \mathbf{d}_{\gamma}^{*}-\varepsilon_{\gamma}^{-1} \mathbf{u}_{\gamma}^{*}\right)\right\|}
$$

At this point, the expression of $\alpha^{*}$ and $\alpha_{\text {Graetz }}$ are analogous. In both cases, the flux of substrate towards the fluid-biofilm interface (which depends on the mass transfer coefficient) is based on a driving force given by the difference between the averaged concentration in the fluid phase $\left\langle c_{A \gamma}\right\rangle^{\gamma}$ and the microscopic concentration at $A_{\gamma \omega}$ (or the concentration value at the tube walls), i.e., 0 .

Consequently, the Sherwood numbers $S h^{*}$ and $S h_{\text {Graetz }}$, associated respectively to $\alpha^{*}$ and $\alpha_{\text {Graetz }}$, can be directly compared. Figure 8 represents the variation of these two numbers as a function of the dimensionless distance (normalized with $R P e$ ) from the inlet.

One can note that the solution of the closure problem is spatially constant while the Graetz solution tends toward an asymptotic value. The MTLC solution is space (and time) invariant due to the assumption of small variations at the microscale of the macroscopic gradient, i.e., nonlocal effects are neglected (see Appendix A). The analytical solution reaches the asymptotic state 
quickly, after a dimensionless distance of $0.11 P e$ only. Consequently, as a function of the position relative to the inlet, one distinguishes two zones:

- An establishment zone, in which the mass transfer coefficient is very sensitive to the Péclet number and to the position. No conventional upscaling is valid here. This behaviour, from an upscaling point of view, could be qualified as non-local, as mentioned above.

- An asymptotic behaviour zone, where the mass transfer coefficient becomes constant (and does not depend on the Péclet number) and inferior to the values obtained in the establishment zone.

The existence of this establishment zone must be kept in mind when using the MTLC model. This fact had raised problems during the validation of the upscaled models by comparison with the direct simulation at the microscale (see section 6.4). Even after the establishment of the asymptotic state, we notice a relative difference of about $15 \%$ between the theoretical Sherwood number (value: 4.75 ) and the analytical Sherwood number (value: 4.07; this is the value of reference). Two main reasons can explain this residual discrepancy:

- In Graetz solution, it is assumed that the diffusion in the $z$-direction can normally be neglected relative to the diffusion in the radial direction (see [16] for an upscaling study of the Graetz problem where this assumption has been made).

- The loss of information between the microscale and the macroscale induced by the volume averaging method, especially by the assumption of small variations of macroscopic variables at the microscale (see Kechagia et al. [25] for a discussion upon the limitations inherent to this assumption). However, this behaviour is in essence specific to the nature of the geometrical case under consideration, i.e., flow in a tube or a stratified system ([16], [35]). 
In conclusion we can say that we have got a reasonably good agreement between the Graetz

solution and the MTLC model solution when the asymptotic state is reached. This comparison also suggests that such a macroscopic model, based on the volume averaging method, is capable of predicting reliable mass transfer coefficients, this by using a single elementary unit cell (whatever its complexity), which is particularly effective.

\subsection{Validity domains of the one-equation non equilibrium macroscopic models}

In order to establish the validity domains associated to the two non-equilibrium one equation models, in terms of hydrodynamic and biochemical conditions of transport (i.e., in a Péclet number - Damköhler number diagram), we adopt the same approach that has been used in Golfier et al. [18] to determine the validity domain of the local mass equilibrium one-equation model (denoted further as the Local Equilibrium Assumption (LEA) model). Results of direct simulations performed at the microscale (computed with COMSOL Multiphysics 3.5) on a simplified porous medium for different values of $P e$ and $D a$ are compared to the equivalent upscaled simulations computed with the macroscopic models under consideration. In our case, we will use a 2D stratified geometry to test the models: the microscale geometry adopted is illustrated in Figure 9. Note that in this paper, the Damköhler number is defined as relative to the thickness of the biofilm, while in Golfier et al. [18] the Damköhler number is defined as a function of the periodicity length of the medium. $\mathcal{D}_{\Gamma}$ has been arbitrarily fixed at 0.25 and $K^{\prime}{ }_{A \omega}$ at 0.5 .

We faced a problem during this comparison. The MTLC model implies strong reaction kinetics (in order to have an instantaneous consumption of the substrate at the fluid-biofilm interface). For too high reaction rates (i.e., a high value of $D a$ ), the solute is totally consumed during its transport through the considered porous medium, so that it is impossible to compare breakthrough curves. We managed this issue by comparing spatial profiles of concentration along the medium at the steady state. This solution leads to other difficulties in the sense that: 
- Non-zero concentration profiles must be longer than the establishment length of the averaged simulations. Otherwise, the problem is non homogenizable. This behaviour appears mainly at low $P e$ and high $D a$. Similarly, this establishment length, which is an increasing function of the Péclet number, must be shorter than the length of the considered porous medium (which was initially fixed to 10, see Figure 9). Thus, for Péclet number values beyond 1000, a porous medium of $30 \mathrm{~L}$ length should be used, in order to reach the asymptotic state before the outlet of the stratified medium.

- Finally, a comparison study restricted to the steady-state regime leads to focus only on the asymptotic behaviour of the macroscopic models although the evaluation of such models in transient conditions may be quite different. The impact of the quasi-steady assumption made in the closure and microscale problems cannot be questioned through this kind of comparison. Such limitations must be kept in mind, especially under high velocity conditions, i.e., at high Péclet number (see [36] for more details). In spite of all these remarks, our validity study was based on the steady solution.

Figure 10 shows three spatial profiles of concentration, which are characteristics of the transition between the validity domains of the MTLC model at low Péclet numbers to the validity domain of RRLC model at high Péclet numbers; all simulations have been performed for a constant high value of the Damköhler number ( $D a=2500$ ).

The comparison of these simulations, collected in Figure 11, suggests that MTLC and RRLC models assumptions require a high enough value of $D a$ to be satisfied. Indeed, the assumption of zero concentration at the fluid-biofilm interface adopted in the MTLC model cannot be verified for a too low reaction. Similarly, the RRLC model is relevant only if concentration gradients do exist in the biofilm phase (a RRLC model with an effectiveness factor of 1 is equivalent to the LEA model with a forced dispersion in the fluid phase). 
One can observe on Figure 11 that the MTLC model is valid at low Pe since in these conditions, with a high enough $D a$, the assumption of a zero concentration at the fluid-biofilm interface is correctly satisfied. On the contrary, the RRLC model is verified at high $P e$ only. The assumption of equality at the interface between the microscale concentration within the biofilm and the fluid averaged concentration requires that the flow velocity is high enough, especially if the Damköhler number is high. Consequently, direct simulations have been performed over a large range of Péclet numbers to correctly determine the appropriate validity domains. Between them, a transition domain appears. The width of this transition domain is an increasing function of $\mathrm{Pe}$ and $\mathrm{Da}$ (at least in the considered configuration). Nevertheless, it must be emphasized that, for Péclet numbers higher than a value of about $10^{3}$, inertial effects should be considered in the calculation of the flow field (the value of the Reynolds number in this case may be equal or higher than 1). This simplification (we have considered only the Stokes equations) probably leads to an underestimation of the limit-value of the Péclet number for the RRLC validity domain, since inertial effects should enhance the mixing of the fluid phase. It should be reminded also that in certain instances (at high velocities mainly), the full transient behavior of the effective parameters can be of interest and consequently, the quasi-steady condition assumed at the microscale might be questionned. As a consequence, the RRLC model should be used in a careful way for highly transient phenomena at high Péclet number.

Figure 11 collects the different simulations performed in a Damköhler number - Péclet number diagram and presents the domains of validity associated to the three one-equation transport models respectively, the LEA model (LEA model results are extracted from [18]), the MTLC model and the RRLC model. Comparisons between the different concentration profiles for each "numerical experiment" have been led by comparing the respective values of their zeroth, first and second order moments.

On the basis of this diagram, several important remarks can be made: 
- Both non-equilibrium models require high values of $D a$. The RRLC model is valid for explained above.

- The validity domain of the RRLC model is particularly restricted and corresponds to very specific conditions (in terms of flow velocity). Given the constraints associated to the Péclet and Damköhler values involved, this formulation is more appropriate to bioengineering applications such as the wastewater biotreatment in packed bed reactors ([50], [51]). An example of practical application will be detailed below. Such a formulation could be considered also for flows in fractured media in groundwater systems [7] where thick (1 to 2 centimeters) biofilms and high velocities are usual. Moreover, this is the more relevant macroscopic one-equation model (compared to the other approaches) for high Damköhler and Péclet numbers and an extended validity domain may be defined, as a function of the level of accuracy required by the user.

- One observes on this figure the presence of a non-homogenisable zone for which the constraint of length scales is not respected anymore (the characteristic length scale associated to the macroscopic concentration gradient is of the same order of magnitude as the characteristic length scales $\ell_{\gamma}$ and $\ell_{\omega}$ ).

Note also that the thresholds and the slopes of the limits between domains are of course highly dependent on the porous medium considered. Results could be slightly different for another kind of pore-scale geometry even if we think that the validity domains would be roughly the same. In conclusion, we can note that with the RRLC and MTLC models, we have two simple models describing non-equilibrium reactive transport in porous media including a biofilm phase. They are well-suited for highly reactive transport conditions. Let us consider, for instance, the problem of nitratification in trickling filters for wastewater treatment studied by [51]. In the nitratification process, Nitrobacter bacteria $\left(\rho_{\omega}=1000 \mathrm{mg} / \mathrm{cm}^{3} ; \mu_{A \omega}=0.55 \mathrm{~d}^{-1}\right.$ and $\left.K_{A \omega}=1.2 \cdot 10^{-3} \mathrm{mg} / \mathrm{cm}^{3}\right)$ 
converts nitrite (coming from the oxidation of ammonia by Nitrosomas) to nitrate $\left(\mathcal{D}_{A \omega}=6 \cdot 10^{-2} \mathrm{~cm}^{2} / \mathrm{d}\right)$ in trickling filters $\left(\ell_{\gamma}=5 \cdot 10^{-1} \mathrm{~cm} ; \ell_{\omega}=1.5 \cdot 10^{-2} \mathrm{~cm}\right)$ used as support medium for biofilm growth. Given the typical flow rates under consideration at the pilot-scale (from 1 to $3 \mathrm{l} / \mathrm{min}$ for a trickling filter of $9 \mathrm{~cm}$ diameter and a porosity of 0.35 ), calculations lead to Péclet and Damköhler values of $10^{5}-10^{6}$ and 1720 , respectively, for which the assumptions of the RRLC model are verified. This practical example illustrates the capacity of such a nonequilibrium model for modeling a real system and addressing the needs of industrial processes in bio-engineering. On the contrary, the MTLC model is well-suited for applications either to very thin biofilms that do not offer significant internal mass transfer barriers and for which the reaction can be assumed to occur at the surface or to multi-population heterogeneous biofilms for which the heterotrophic activity is limited to the biofilm surface ([9], [20]). Another potential application would be for the modeling of transport of antimicrobial agents such as oxidizing biocides chlorine or hydrogen peroxide which are known to be neutralized in the surface layers of the biofilm faster than they diffuse into the biofilm ([44], [46])

Finally, a zone subsists where all one equation models fail and for which a two-equation description is required in order to have an accurate simulation of transport phenomena. The development of such a model will be the focus of a further study.

\section{Summary and conclusion}

In this study we have presented the equations describing bioreactive transport in a porous medium at the Darcy-scale for two non equilibrium one-equation models. The macroscopic form of these two models, the Reaction Rate Limited Consumption model and the Mass Transport Limited Consumption model, is reminded below:

\section{RRLC model}




$$
\begin{aligned}
& \frac{\partial\left(\varepsilon_{\gamma}\left\langle c_{A \gamma}\right\rangle^{\gamma}\right)}{\partial t}+\nabla \cdot\left(\varepsilon_{\gamma}\left\langle\mathbf{v}_{\gamma}\right\rangle^{\gamma}\left\langle c_{A \gamma}\right\rangle^{\gamma}\right)=\nabla \cdot\left(\mathbf{D}_{A, \text { eff }}^{*} \cdot \nabla\left\langle c_{A \gamma}\right\rangle^{\gamma}\right) \\
& +\nabla \cdot\left(\mathbf{d}_{\gamma}^{*}\left\langle c_{A \gamma}\right\rangle^{\gamma}\right)+\left(\mathbf{u}_{\gamma}^{*}-\mathcal{D}_{A \gamma} \nabla \varepsilon_{\gamma}\right) \cdot \nabla\left\langle c_{A \gamma}\right\rangle^{\gamma}-\alpha\left\langle c_{A \gamma}\right\rangle^{\gamma}
\end{aligned}
$$

\section{MTLC model}

If such formulations have already been introduced heuristically in the past, the originality of this approach lies in the fact that it keeps a quantitative description of the coupled nature of transport and reaction and therefore provides a rigorous formalism for the expression of interfacial fluxes. Under the condition that the assumptions of the considered models are verified, we can evaluate the effective parameters (dispersion, interfacial fluxes ...) from solving pore-scale closure problems. These problems require the knowledge of some pore-scale characteristics, and this would probably call for some specific laboratory measurements. In practice, these transport models, which require to be coupled with a biofilm growth model if we are interested in studying the complete process of biodegradation, will possibly be applied to many systems of interest by extending the classical domain of application of one-equation models to non-equilibrium conditions.

The validity domain of each non-equilibrium transport model has been assessed in terms of hydrodynamic and biochemical conditions of transport (i.e., in terms of $P e$ and $D a$ numbers). The RRLC model is suitable for high Péclet and high Damköhler numbers (because these conditions enhance the homogeneity of the concentration within the fluid phase and the formation of concentration gradients in the biofilm), while the MTLC model assumptions are verified for low Péclet numbers and high Damköhler numbers (conditions for which the 
concentration field in the fluid phase tends to satisfy the assumption of zero concentration at the biofilm-fluid interface).

Further numerical experiments would be required in order to investigate the impact of the microscopic features of the problem on the macroscopic models behaviour; in particular it would be interesting to make computations on a realistic pore-scale geometry (see [18]). A later paper will develop these points. For transport conditions located out of the validity domains of LEA, RRLC and MTLC models, a general two equation model should be established. The development of such a model will be the goal of further studies.

\section{Acknowledgement}

This work has been partially supported by the EC2CO - CNRS/INSU program and the French Scientific Interest Group - Industrial Wasteland (GISFI).

\section{References}

[1] A. Ahmadi, M. Quintard, S. Whitaker, Transport in chemically and mechanically heterogeneous porous media V: Two-equation model for solute transport with adsorption, Adv. Water Resour. 22 (1998) 59-86.

[2] B. Atkinson and I. J. Davies, The overall rate of substrate uptake (reaction) by microbial films I: Biological rate equation, Trans. Inst. Chem. Engr. 52 (1974) 248-259.

[3] K.H. Baker and D.S. Herson, In situ Bioremediation of Contaminated Aquifers and Subsurface Soils, Geomicrobiology Journal 8 (1990) 133-146.

[4] P. Baveye and A. Valocchi, An evaluation of mathematical models of the transport of biologically reacting solutes in saturated soils and aquifers, Water Resour. Res. 25(6) (1989) 1413-1421.

[5] R. Bibby, Mass transport of solutes in dual-porosity media, Water Resour. Res. 17(4) (1981) 1075-1081.

[6] H.W. Blanch and D.S. Clark, Biochemical Engineering, Marcel Dekker Ltd, 1996.

[7] A. Charbonneau, K. Novakowski, , N. Ross, The effect of a biofilm on solute diffusion in fractured porous media, J. Contam. Hydrol. 85(3-4) (2006) 212-228.

[8] F. Cherblanc, A. Ahmadi, M. Quintard, Two-domain description of solute transport in heterogeneous porous media: Comparison between theoretical predictions and numerical experiments, Adv. Water Resour. 30 (2007) 1127-1143.

[9] A.C. Cole, M.J. Semmens, T.M. LaPara, Stratification of activity and bacterial community structure in biofilms grown on membranes transferring oxygen, Appl. Environ. Microbiol. 70(4) (2004) 1982-1989.

[10] M. Farhadian, C. Vachelard, D. Duchez, C. Larroche, In situ bioremediation of 
monoaromatic pollutants in groundwater: A review, Bioresource Technology 99 (2008) 52965308.

[11] H.S. Fogler, Elements of Chemical Reaction Engineering, 3rd edition, Prentice Hall, 1999

[12] D.J. Gapes and J. Keller, Impact of oxygen mass transfer on nitrification reactions in suspended carrier reactors biofilms, Process. Biochem. 44 (2009) 43-53.

[13] R. Glowinski, Numerical methods for Nonlinear Variational Problems, Springer Verlag: New York, NY, USA, 1984.

[14] F. Golfier, Dissolution des roches carbonatées par injection d'acide, $\mathrm{PhD}$ thesis, Institut National Polytechnique de Toulouse, 2001.

[15] F. Golfier, C. Zarcone, B. Bazin, R. Lenormand, D. Lasseux, M. Quintard, On the ability of a Darcy-scale model to capture wormhole formation during the dissolution of a porous medium, J. Fluid Mech., 457 (2002) 213-254.

[16] F. Golfier, M. Quintard, S. Whitaker, Heat and Mass Transfer in Tubes: An Analysis Using the Method of Volume Averaging, J. Porous Media, 5 (2002b) 169-85.

[17] F. Golfier, M. Quintard, F. Cherblanc, B. Zinn, B. Wood, Comparison of theory and experiment for solute transport in highly heterogeneous porous medium, Adv. Water Resour. 30 (2007) 2235-2261.

[18] F. Golfier, B. Wood, L. Orgogozo, M. Quintard, M.A. Buès, Biofilms in Porous Media: Development of Macroscopic Transport Equations via Volume Averaging with Closure for Local Mass Equilibrium Conditions, Adv. Water Resour. 32(3) (2009) 463-485.

[19] W.G. Gray, A. Leijnse, R.L. Kolar, C.A. Blain, Mathematical Tools for Changing Spatial Scales in the Analysis of Physical Systems, CRC Press: Boca Raton, FL, 1993.

[20] H. Horn, D.C. Hempel, Growth and decay in an auto-/heterotrophic biofilm, Water Res, 31(9) (1997) 2243-2252.

[21] F.A. Howes, S. Whitaker, The spatial averaging theorem revisited, Chem. Eng. Sci. 40 (1985) 1387-1392.

[22] J.R. Hunt, P.A. Holden, M.K. Firestone, Coupling Transport and Biodegradation of VOCs in Surface and Subsurface Soils, Environ. Health Perspect. 103(5) (1995) 75-78.

[23] M. Jakob, Heat Transfer, John Wiley \& Sons, Inc., New York, 1949.

[24] P.A. Jennings, V.L. Snoeyink, E.S.K. Chian, Theoretical model for a submerged biological filter, Biotechnol. Bioeng. 18 (1976) 1249-1273.

[25] P.E. Kechagia, I.N. Tsimpanogiannis, Y.C. Yortsoso, P.C. Lischtner, On the upscaling of reaction-transport processes in porous media with fast or finite kinetics, Chem. Eng. Sci. 57 (2001) 2565-2577.

[26] J.S. Kindred, M.A. Celia, Contaminant transport and biodegradation, 2, Conceptual model and test simulations, Water Resour. Res., 25(6) (1989) 1149-1159.

[27] K.S.W. Kirchner and H.J. Rehm, Exhaust gas purification using biocatalysts (fixed bacteria monocultures $)$ - the influence of biofilm diffusion rate $\left(\mathrm{O}_{2}\right)$ on the overall reaction rate, Appl Microbiol. Biotechnol. 37 (1992) 277-279.

[28] F.E. Löffler and E.A. Edwards, Harnessing microbial activities for environmental cleanup, Curr. Opin. Biotechnol. 17 (2006) 274-284.

[29] W.J. Maier, V.C. Behn, C.D. Gates, Simulation of the trickling filter process, J. Sanit. Eng. Divn. ASCE 93 (1967) 91-112.

[30] R.D. Megee, S. Kinoshita, A.G. Fredrickson, H.M. Tsuchiya, Differentiation and product formation in molds, Biotechnol. Bioeng. 12 (1970) 771-801.

[31] H. Mulder, A.M. Breure, W.H. Rulkens, Prediction of complete bioremediation periods for PAH soil pollutants in different physical states by mechanistic models, Chemosphere 43(8) (2001) 1085-1094.

[32] National Research Council, In situ bioremediation: When does it work?. National Academy Press, Washington DC, 1993. 
[33] J.A. Ochoa-Tapia, P. Stroeve, S. Whitaker, Diffusive transport in two-phase media: Eng. Sci. 49 (1994) 709-726.

[34] C. Picioreanu, M.C.M. Van Loosdrecht, J.J. Heijnen, Effect of diffusive and convective substrate transport on biofilm structure formation: a two-dimensional modeling study, Biotechnol Bioeng. 69(5) (2000) 504-515.

[35] C. Pierre, F. Plouraboué, M. Quintard, Convergence of generalized volume averaging method on a convection-diffusion problem: a spectral perspective, SIAM J. App. Math 66(1) (2006) 122-152.

[36] M. Quintard, S. Whitaker, One- and two-equation models for transient diffusion processes in two-phase system, Advances in Heat Transfer 23 (1993) 369-465.

[37] M. Quintard, Diffusion in isotropic and anisotropic porous systems. Transport in Porous Media, 11 (1993) 187-199.

[38] M. Quintard, S. Whitaker, Convection, dispersion, and interfacial transport of contaminants: homogeneous porous media, Adv. Water Resour. 17 (1994) 221-239.

[39] M. Quintard, M. Kaviany, S. Whitaker, Two-medium treatment of heat transfer in porous media: Numerical results for effective properties, Adv.Water Resour. 20 (1997) 77-94.

[40] P.S.C. Rao, D.E. Rolston, R.E. Jessup, J.M. Davidson, Solute transport in aggregated porous media: Theoretical and experimental evaluation, Soil Sci. Am. J. 44(6) (1980)1139-1146.

[41] P. Rasetarinera, Etude Mathématique et Numérique de la Restauration Biologique En Milieu Poreux, PhD thesis, Université de Bordeaux 1, 1995.

[42] W.F. Ritter and R.W. Scarborough, A Review of Bioremediation of Contaminated Soils and Groundwater, Journal of Environmental Science and Health Part a-Environmental Science and Engineering \& Toxic and Hazardous Substance Control 30 (1995) 333-357.

[43] B.E. Rittmann and P.L. McCarty, Substrate flux into biofilms of any thickness, J. Environ. Engr. 107 (1981) 831-849.

[44] M.E. Roberts, P.S. Stewart, Modelling protection from antimicrobial agents in biofilms through the formation of persister cells, Microbiol. 151 (2005) 75-80.

[45] W.F.M. Roling and H.W. van Verseveld, Natural attenuation: What does the subsurface have in store?, Biodegradation 13 (2002) 53-64.

[46] P.S. Stewart, G.A. McFeters, C.T. Huang, Biofilm control by antimicrobial agents. In Biofilms II: Process analysis and applications. Edited by J.D. Bryers., New York:Wiley-Liss., 2000, pp. 373-405.

[47] P.J. Sturman, P.S. Stewart, A.B. Cunningham, E.J. Bouwer, J.H. Wolfram, Engineering Scale-up of in-Situ Bioremediation Processes - a Review, Journal of Contaminant Hydrology 19 (1995) 171-203.

[48] G.I. Taylor, Dispersion of soluble matter in solvent flowing slowly through a tube, Proc. Royal Soc. A. 219 (1953) 186-203.

[49] H. Van der Vorst, Bi-CGSTAB: A fast and smoothly converging variant of Bi-CG for the solution of nonsymmetric linear systems, SIAM J. Sci. Statist. Comput., 13 (1992) 631-644. [50] D.V. Vayenas, G. Lyberatos, On the design of nitrifying trickling filters for potable water treatment, Wat. Res., 29(4) (1995) 1079-1084.

[51] D.V. Vayenas, S. Pavlou, G. Lyberatos, Development of a dynamic model describing nitrification and nitratification in trickling filters, Water Res. 31(5) (1997) 1135-1147.

[52] J. D. Wall and L.R. Krumholz, Uranium reduction, Annual Review of Microbiology 60 (2006) 149-166.

[53] J.V. Weiss and I.M. Cozzarelli, Biodegradation in contaminated aquifers: Incorporating microbial/molecular methods, Ground Water 46 (2008) 305-322.

[54] S. Whitaker, Introduction to fluid mechanics. Malabar, FL, USA: R.E. Krieger, 1981. 
[55] S. Whitaker, The method of volume averaging, Dordrecht: Kluwer Academic Publishers, 1999.

[56] E.J. Wolfrum, A.S. Watt, Bioreactor design studies for a Hydrogen-producing bacterium, Appl Biochem Biotechnol, 98-100 (2002) 611-625.

[57] B.D. Wood, S. Whitaker, Diffusion and reaction in biofilms, Chem. Engng. Sci. 53(3) (1998) 397-425.

[58] B.D. Wood, S. Whitaker, Cellular growth in biofilms, Biotechnol. Bioeng. 64 (1999) 656670.

[59] B.D. Wood, S. Whitaker, Multi-species diffusion and reaction in biofilms and cellular media. Chem. Engng. Sci. 55 (2000) 3397-3418.

[60] B.D. Wood, M. Quintard, S. Whitaker, Calculation of effective diffusivities for biofilms and tissues, Biotechnol. Bioeng. 77(5) (2002) 495-516.

[61] B.D. Wood, F. Golfier, and M. Quintard, Dispersive Transport in Porous Media with Biofilm: Local Mass Equilibrium in Simple Unit Cells, Int. J. of Environment and Waste Management (2008 in press)

[62] B.D. Wood, The role of scaling laws in upscaling, Adv. Water Resour. 32 (5) (2009) 723 736.

\section{Appendixes}

\section{Appendix A. Development of closure problems for the RRLC model}

If we consider the equality:

$$
\tilde{c}_{A \gamma}=c_{A \gamma}-\left\langle c_{A \gamma}\right\rangle^{\gamma}
$$

and by using the following rearranged form of the non-closed equation of transport in the fluid phase, Eq. (16),

$$
\begin{aligned}
& \frac{\partial\left\langle c_{A \gamma}\right\rangle^{\gamma}}{\partial t}+\left\langle\mathbf{v}_{\gamma}\right\rangle^{\gamma} \cdot \nabla\left\langle c_{A \gamma}\right\rangle^{\gamma}=\nabla \cdot\left(\mathcal{D}_{A \gamma} \nabla\left\langle c_{A \gamma}\right\rangle^{\gamma}\right) \\
& +\varepsilon_{\gamma}{ }^{-1} \nabla \varepsilon_{\gamma} \cdot\left(\mathcal{D}_{A \gamma} \nabla\left\langle c_{A \gamma}\right\rangle^{\gamma}\right)+\varepsilon_{\gamma}{ }^{-1} \nabla \cdot\left[\varepsilon_{\gamma} \mathcal{D}_{A \gamma}\left(\frac{1}{V_{\gamma}} \int_{A_{\omega \gamma}(t)} \mathbf{n}_{\gamma \omega} \tilde{c}_{A \gamma} d A+\frac{1}{V_{\gamma}} \int_{A_{\gamma k}(t)} \mathbf{n}_{\gamma \kappa} \tilde{c}_{A \gamma} d A\right)\right] \\
& +\frac{1}{V_{\gamma}} \int_{A_{\omega \gamma}(t)} \mathbf{n}_{\gamma \omega} \cdot\left(\mathcal{D}_{A \gamma} \nabla c_{A \gamma}\right) d A+\frac{1}{V_{\gamma}} \int_{A_{\gamma \kappa}(t)} \mathbf{n}_{\gamma \kappa} \cdot\left(\mathcal{D}_{A \gamma} \nabla c_{A \gamma}\right) d A \\
& -\varepsilon_{\gamma}{ }^{-1} \nabla \cdot\left\langle\tilde{\mathbf{v}}_{\gamma} \tilde{c}_{A \gamma}\right\rangle-\varepsilon_{\gamma}{ }^{-1}\left\langle c_{A \gamma}\right\rangle^{\gamma} \frac{\partial\left(\varepsilon_{\gamma}\right)}{\partial t}
\end{aligned}
$$

we can obtain the following relation by subtracting Eq. (A.2) to the point equation of transport, 
Eq. (2):

$$
\begin{aligned}
\frac{\partial \tilde{c}_{A \gamma}}{\partial t}=\nabla & \cdot\left(\mathcal{D}_{A \gamma} \nabla \tilde{c}_{A \gamma}\right)-\mathbf{v}_{\gamma} \cdot \nabla \tilde{c}_{A \gamma}-\tilde{\mathbf{v}}_{\gamma} \cdot \nabla\left\langle c_{A \gamma}\right\rangle^{\gamma}-\left(\varepsilon_{\gamma}^{-1} \nabla \varepsilon_{\gamma}\right) \cdot\left(\mathcal{D}_{A \gamma} \nabla\left\langle c_{A \gamma}\right\rangle^{\gamma}\right) \\
& -\varepsilon_{\gamma}^{-1} \nabla \cdot\left[\varepsilon_{\gamma} \mathcal{D}_{A \gamma}\left(\frac{1}{V_{\gamma}} \int_{A_{\omega \gamma}(t)} \mathbf{n}_{\gamma \omega} \tilde{c}_{A \gamma} d A+\frac{1}{V_{\gamma}} \int_{A_{\gamma \kappa}(t)} \mathbf{n}_{\gamma \kappa} \tilde{c}_{A \gamma} d A\right)\right] \\
& -\frac{1}{V_{\gamma}} \int_{A_{\omega \gamma}(t)} \mathbf{n}_{\gamma \omega} \cdot\left(\mathcal{D}_{A \gamma} \nabla c_{A \gamma}\right) d A-\frac{1}{V_{\gamma}} \int_{A_{\gamma \kappa}(t)} \mathbf{n}_{\gamma \kappa} \cdot\left(\mathcal{D}_{A \gamma} \nabla c_{A \gamma}\right) d A \\
& +\varepsilon_{\gamma}^{-1} \nabla \cdot\left\langle\tilde{\mathbf{v}}_{\gamma} \tilde{c}_{A \gamma}\right\rangle+\varepsilon_{\gamma}^{-1}\left\langle c_{A \gamma}\right\rangle^{\gamma} \frac{\partial\left(\varepsilon_{\gamma}\right)}{\partial t}
\end{aligned}
$$

At this point, the use of the following geometric lemma [38]:

$$
\frac{1}{V} \int_{A_{\omega \gamma}(t)} \mathbf{n}_{\gamma \omega} d A+\frac{1}{V} \int_{A_{\omega \kappa}(t)} \mathbf{n}_{\gamma K} d A=-\nabla \varepsilon_{\omega}
$$

leads to the following expression of the deviation equation:

$$
\begin{aligned}
\frac{\partial \tilde{c}_{A \gamma}}{\partial t}=\nabla & \cdot\left(\mathcal{D}_{A \gamma} \nabla \tilde{c}_{A \gamma}\right)-\mathbf{v}_{\gamma} \cdot \nabla \tilde{c}_{A \gamma}-\tilde{\mathbf{v}}_{\gamma} \cdot \nabla\left\langle c_{A \gamma}\right\rangle^{\gamma} \\
& -\varepsilon_{\gamma}^{-1} \nabla \cdot\left[\varepsilon_{\gamma} \mathcal{D}_{A \gamma}\left(\frac{1}{V_{\gamma}} \int_{A_{\omega \gamma}(t)} \mathbf{n}_{\gamma \omega} \tilde{c}_{A \gamma} d A+\frac{1}{V_{\gamma}} \int_{A_{\gamma \kappa}(t)} \mathbf{n}_{\gamma \kappa} \tilde{c}_{A \gamma} d A\right)\right] \\
& -\frac{1}{V_{\gamma}} \int_{A_{\omega \gamma}(t)} \mathbf{n}_{\gamma \omega} \cdot\left(\mathcal{D}_{A \gamma} \nabla \tilde{c}_{A \gamma}\right) d A-\frac{1}{V_{\gamma}} \int_{A_{\gamma \kappa}(t)} \mathbf{n}_{\gamma \kappa} \cdot\left(\mathcal{D}_{A \gamma} \nabla \tilde{c}_{A \gamma}\right) d A \\
& +\varepsilon_{\gamma}{ }^{-1} \nabla \cdot\left\langle\tilde{\mathbf{v}}_{\gamma} \tilde{c}_{A \gamma}\right\rangle+\varepsilon_{\gamma}{ }^{-1}\left\langle c_{A \gamma}\right\rangle^{\gamma} \frac{\partial\left(\varepsilon_{\gamma}\right)}{\partial t}
\end{aligned}
$$

Now, let us simplify Eq. (A.3) by making an order-of-magnitude analysis of the different terms and by using the hypothesis of separation of scales [38]. Theses estimates lead to:

$$
\begin{gathered}
\mathbf{v}_{\gamma} \cdot \nabla \tilde{c}_{A \gamma} \gg \nabla \cdot\left\langle\tilde{\mathbf{v}}_{\gamma} \tilde{c}_{A \gamma}\right\rangle \\
\nabla \cdot\left(\mathcal{D}_{A \gamma} \nabla \tilde{c}_{A \gamma}\right) \gg \varepsilon_{\gamma}^{-1} \nabla \cdot\left[\varepsilon_{\gamma} \mathcal{D}_{A \gamma}\left(\frac{1}{V_{\gamma}} \int_{A_{\omega \gamma}(t)} \mathbf{n}_{\gamma \omega} \tilde{c}_{A \gamma} d A+\frac{1}{V_{\gamma}} \int_{A_{\gamma k}(t)} \mathbf{n}_{\gamma \kappa} \tilde{c}_{A \gamma} d A\right)\right] \\
\nabla \cdot\left(\mathcal{D}_{A \gamma} \nabla \tilde{c}_{A \gamma}\right) \gg \frac{\partial \tilde{c}_{A \gamma}}{\partial t}
\end{gathered}
$$


Some of the terms neglected in the above estimates (Eq. (A.6.a) and Eq. (A.6.b)) are identified as non-local terms, i.e., for which the spatial deviation concentration is evaluated at points other than the centroid of the averaging volume. These terms are averaged quantities, of which the order of magnitude is $\odot\left(\frac{1}{L}\right)$ whereas the other terms are the order of $\odot\left(\frac{1}{l_{\gamma}}\right)$ which leads to neglect them. At last, the time derivatives are neglected and the problem is treated as quasi-steady since a separation of time scales can be reasonably assumed (see [36] for more details). When the simplifications stated above are adopted, the deviation equation becomes

$$
\begin{aligned}
\mathbf{v}_{\gamma} \cdot \nabla \tilde{c}_{A \gamma}+\tilde{\mathbf{v}}_{\gamma} \cdot \nabla\left\langle c_{A \gamma}\right\rangle^{\gamma}=\nabla \cdot\left(\mathcal{D}_{A \gamma} \nabla \tilde{c}_{A \gamma}\right) \\
\quad-\frac{1}{V_{\gamma}} \int_{A_{\theta \gamma}(t)} \mathbf{n}_{\gamma \omega} \cdot\left(\mathcal{D}_{A \gamma} \nabla \tilde{c}_{A \gamma}\right) d A-\frac{1}{V_{\gamma}} \int_{A_{\mu \kappa}(t)} \mathbf{n}_{\gamma \kappa} \cdot\left(\mathcal{D}_{A \gamma} \nabla \tilde{c}_{A \gamma}\right) d A
\end{aligned}
$$

B.C.1

$$
-\mathbf{n}_{\gamma \kappa} \cdot \mathcal{D}_{A \gamma} \nabla \tilde{c}_{A \gamma}=\mathbf{n}_{\gamma \kappa} \cdot \mathcal{D}_{A \gamma} \nabla\left\langle c_{A \gamma}\right\rangle^{\omega}, \text { at } A_{\gamma \kappa}
$$

B.C. 2

$$
\tilde{c}_{A \gamma}=0, \text { at } A_{\gamma}
$$

The last boundary condition is imposed by the assumption $c_{A \omega}=K_{A, e q}\left\langle c_{A \gamma}\right\rangle^{\gamma}$, at $A_{\gamma \omega}$ of the RRLC model. In order to close the problem, we must find a means of relating the perturbation concentration $\tilde{c}_{A \gamma}$ in terms of the macroscopic source term $\nabla\left\langle c_{A \gamma}\right\rangle^{\gamma}$. We inject the closure assumption,

$$
\tilde{c}_{A \gamma}=\mathbf{b}_{A \gamma} \cdot \nabla\left\langle c_{A \gamma}\right\rangle^{\gamma}
$$

in the former equation. An important assumption is made at this point: it is the assumption of small variations at the microscale of macroscopic concentration gradients (see Kechagia et al. [25] for an analysis of limitations of such an assumption), which allows writing:

$$
\nabla \tilde{c}_{A \gamma}=\nabla\left\langle c_{A \gamma}\right\rangle^{\gamma} \cdot \nabla \mathbf{b}_{A \gamma}
$$


And if we consider now $\varepsilon_{\gamma}$ as a spatially constant quantity, we can finally obtain the closure problem:

$$
\begin{aligned}
\mathbf{v}_{\gamma} \cdot \nabla \mathbf{b}_{A \gamma}+\tilde{\mathbf{v}}_{\gamma} & =\nabla \cdot\left(\mathcal{D}_{A \gamma} \nabla \mathbf{b}_{A \gamma}\right) \\
& -\frac{1}{V_{\gamma}} \int_{A_{\omega \gamma}(t)} \mathbf{n}_{\gamma \omega} \cdot \mathcal{D}_{A \gamma} \nabla \mathbf{b}_{A \gamma} d A-\frac{1}{V_{\gamma}} \int_{A_{\gamma k}(t)} \mathbf{n}_{\gamma \kappa} \cdot \mathcal{D}_{A \gamma} \nabla \mathbf{b}_{A \gamma} d A
\end{aligned}
$$

B.C.1

$$
-\mathbf{n}_{\gamma \kappa} \cdot \nabla \mathbf{b}_{A \gamma}=\mathbf{n}_{\gamma \kappa}, \quad \text { at } A_{\gamma \kappa}
$$

B.C.2

$$
\mathbf{b}_{A \gamma}=0, \text { at } A_{\gamma \omega}
$$

B.C.3

$$
\mathbf{b}_{A \gamma}=\mathbf{b}_{A \gamma}\left(\mathbf{r}+\mathbf{I}_{i}\right), \quad i=1,2,3, \quad \text { at } A_{\gamma e}
$$

B.C.3

$$
\left\langle\mathbf{b}_{A \gamma}\right\rangle^{\gamma}=0
$$

where periodicity conditions and constraints of zero averages by phase have been added to close the problem.

Appendix B. Calculation of the effectiveness factor.

First, we start from the following expression of the interfacial flux between the $\gamma$-phase and the $\omega$ phase:

$$
\begin{aligned}
\frac{1}{V} \int_{A_{\omega \gamma}(t)} \mathbf{n}_{\gamma \omega} \cdot\left(\mathcal{D}_{A \gamma} \nabla c_{A \gamma}\right) d A & =\frac{1}{V} \int_{A_{\omega \gamma}(t)} \mathbf{n}_{\gamma \omega} \cdot\left(\mathbf{D}_{A \omega} \cdot \nabla c_{A \omega}\right) d A \\
& =\frac{K_{A, e q}^{-1}\left\langle c_{A \gamma}\right\rangle^{\gamma}}{V} \int_{A_{\omega \gamma}(t)} \mathbf{n}_{\gamma \omega} \cdot\left(\mathbf{D}_{A \omega} \cdot \nabla S\right) d A
\end{aligned}
$$

Under the quasi-steady state assumption for mass exchange between phases at the microscale, one can consider that the interfacial flux and the value of the biodegradation rate integrated on the biofilm phase are equal. Then, we define the effectiveness factor as the ratio of the real biodegradation rate and the maximum biodegradation rate, which should be observed if the whole biofilm phase was exposed to the concentration available at the fluid-biofilm interface. 
Thus, the effectiveness factor $\eta$ can be expressed as:

$$
\begin{aligned}
\eta= & \frac{\text { Observed effective reaction rate }}{\text { Maximal effective reaction rate }} \\
= & \frac{\frac{1}{V} \int_{A_{\theta \gamma}(t)} \mathbf{n}_{\gamma \omega} \cdot\left(\mathbf{D}_{A \omega} \cdot \nabla c_{A \omega}\right) d A}{\frac{1}{V} \int_{V_{\omega}} \mu_{A \omega} \rho_{\omega} \frac{K_{A, e q}^{-1}\left\langle c_{A \gamma}\right\rangle^{\gamma}}{K_{A, e q}^{-1}\left\langle c_{A \gamma}\right\rangle^{\gamma}+K_{A \omega}} \frac{\left\langle c_{B \omega}\right\rangle^{\omega}}{\left\langle c_{B \omega}\right\rangle^{\omega}+K_{B \omega}} d V}
\end{aligned}
$$

Note that this effectiveness factor is related to the consumption of the substrate A; a similar effectiveness factor can be defined for the electron acceptor B.

From this definition, we can rewrite the interfacial flux as follows:

$$
\frac{1}{V} \int_{A_{\omega \gamma}(t)} \mathbf{n}_{\gamma \omega} \cdot\left(\mathbf{D}_{A \omega} \cdot \nabla c_{A \omega}\right) d A=\eta \varepsilon_{\omega} \mu_{A \omega} \rho_{\omega} \frac{K_{A, e q}^{-1}\left\langle c_{A \gamma}\right\rangle^{\gamma}}{K_{A, e q}^{-1}\left\langle c_{A \gamma}\right\rangle^{\gamma}+K_{A \omega}} \frac{\left\langle c_{B \omega}\right\rangle^{\omega}}{\left\langle c_{B \omega}\right\rangle^{\omega}+K_{B \omega}}
$$

If we transform the expression of effectiveness factor into a dimensionless expression, we obtain:

$$
\eta=\frac{\left(K_{e f f}+1\right)}{c_{B \omega}^{*} \varepsilon_{\omega} K_{e f f} D a} \int_{A_{\gamma \omega}} \mathbf{n}_{\gamma \omega} \cdot \nabla S d A
$$

where the following dimensionless variables and parameters have been defined by:

$$
\begin{gathered}
D a=\frac{\ell_{\omega}^{2} \mu_{A \omega} \rho_{\omega}}{\mathcal{D}_{A \omega} K_{A \omega}} \\
K_{e f f}=\frac{K_{A \omega}}{\left\langle c_{A \gamma}\right\rangle^{\gamma} K_{A, e q}^{-1}} \\
c_{B \omega}^{*}=\frac{\left\langle c_{B \omega}\right\rangle^{\omega}}{\left\langle c_{B \omega}\right\rangle^{\omega}+K_{B \omega}}
\end{gathered}
$$

Here, $\mathcal{D}_{A \omega}$ is the current component of the diffusion tensor in the $\omega$-phase, supposed to be spherical.

\section{List of Figures}


Figure 1: Characteristic length scales and levels associated with porous-medium-supported biofilms.

Figure 2: Characteristic length scales associated with the averaging volume, $\mathcal{V}$, defined at the pore scale.

Figure 3: Conceptual representation of the concentration evolution at the fluid/biofilm interface

Figure 4: Example of a 2D unit cell

Figure 5: Pore-scale conceptual model of a porous medium with biofilm

Figure 6: Comparison between the theoretical calculation and the analytical solution of the effectiveness factor in the case of first order kinetics of reaction.

Figure 7: Illustration of the geometry and boundary conditions associated to the Graetz problem.

Figure 8: Comparison between the Graetz semi-analytical solution and the upscaled solution of the evolution of the Sherwood number along an axi-symmetric tube

Figure 9: Illustration of the geometry and boundary conditions of the stratified system adopted for direct numerical simulations.

Figure 10: Spatial profiles of concentration at steady-state along a stratified porous medium including a biofilm phase. The different concentration profiles are calculated by direct 
simulations at the pore scale, LEA model, MTLC model and RRLC model, with $D a=2500$ and $P e=10^{-2}, 10,10^{3}$.

Figure 11: The domains of validity of the one-equation models of bio-reactive solute transport in porous media with biofilm, in a Damköhler number - Péclet number diagram. 


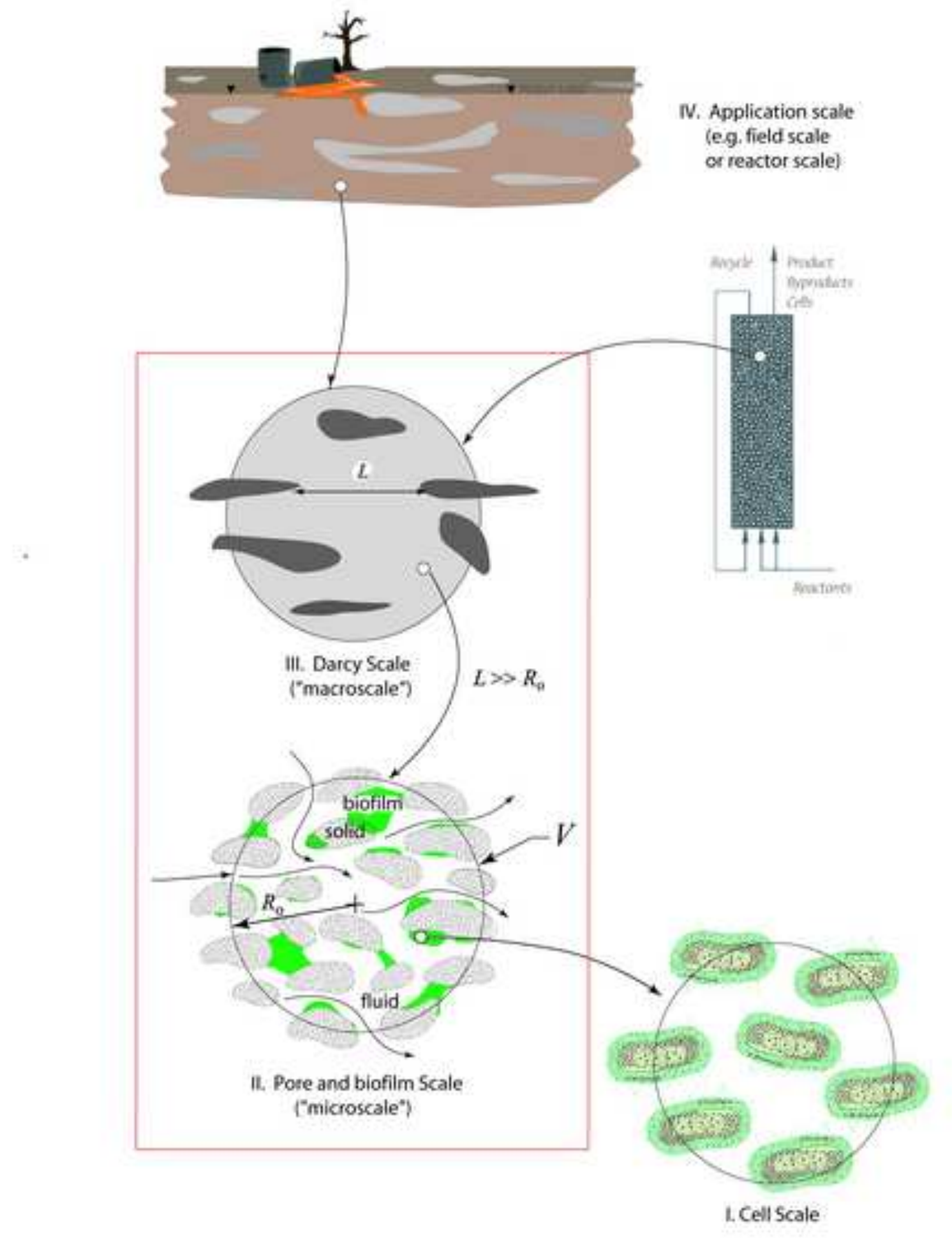
(e.g. field scale cr reactor scale) 


\section{Figure 2}

Click here to download high resolution image

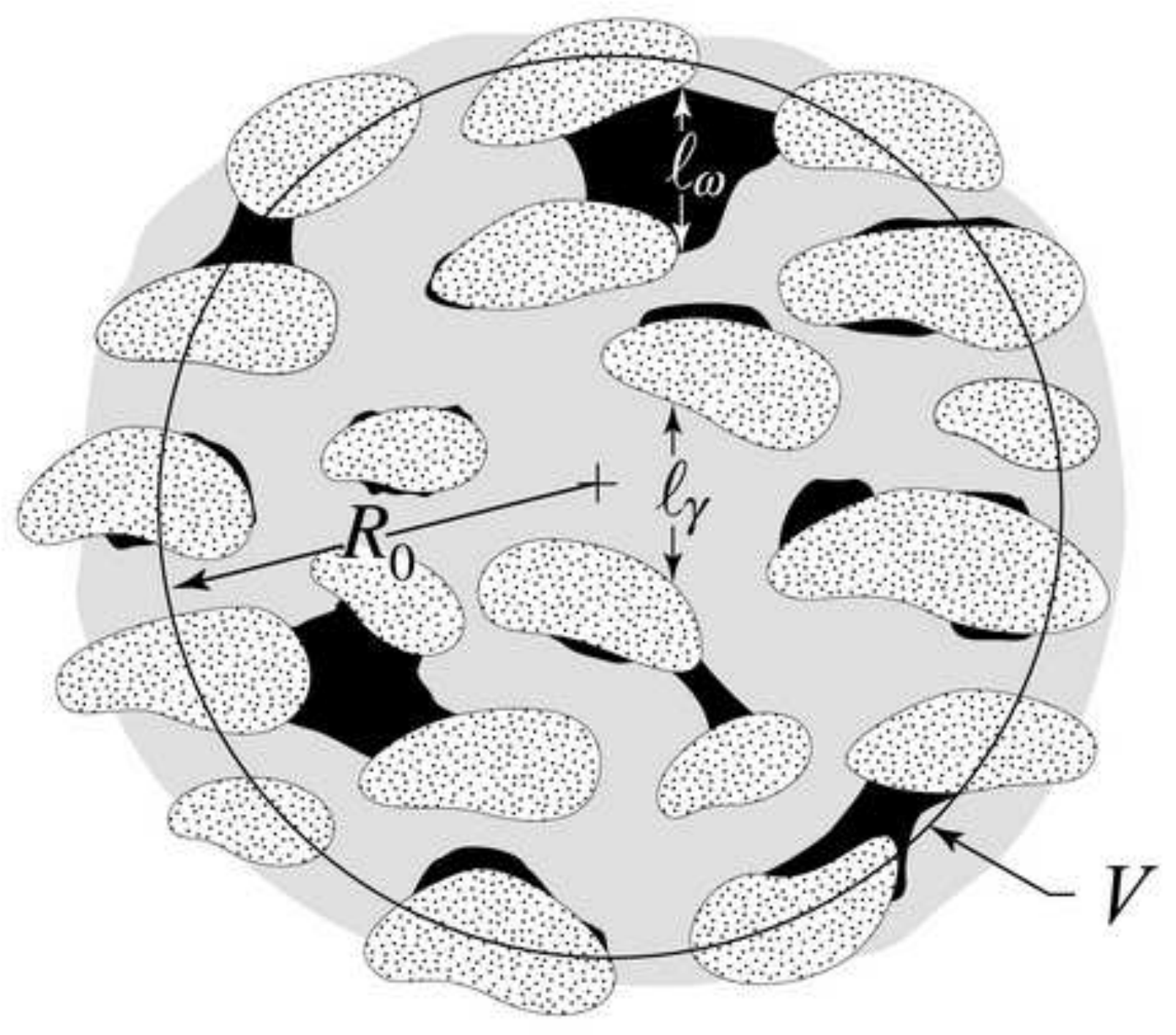




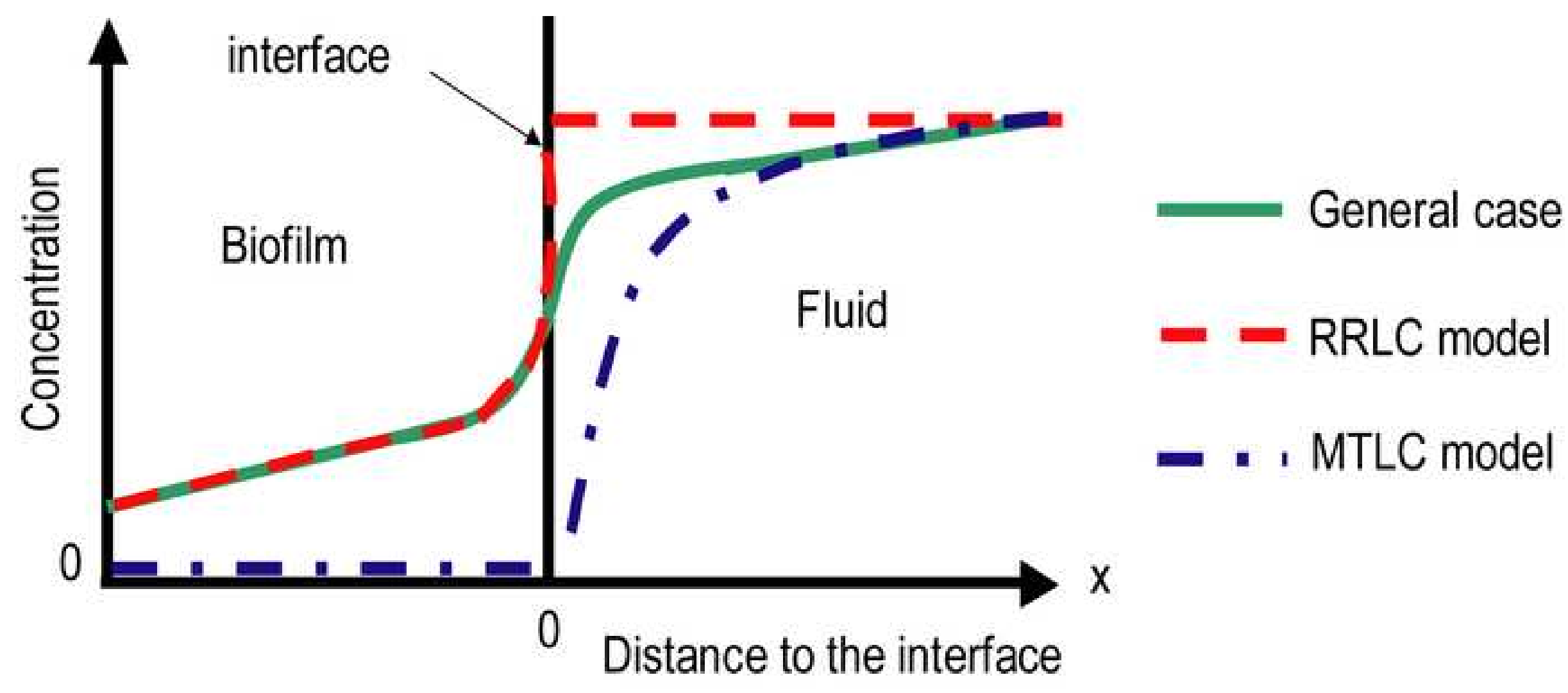


Figure 4
Click here to download high resolution image

fluid ( $\gamma$-phase)

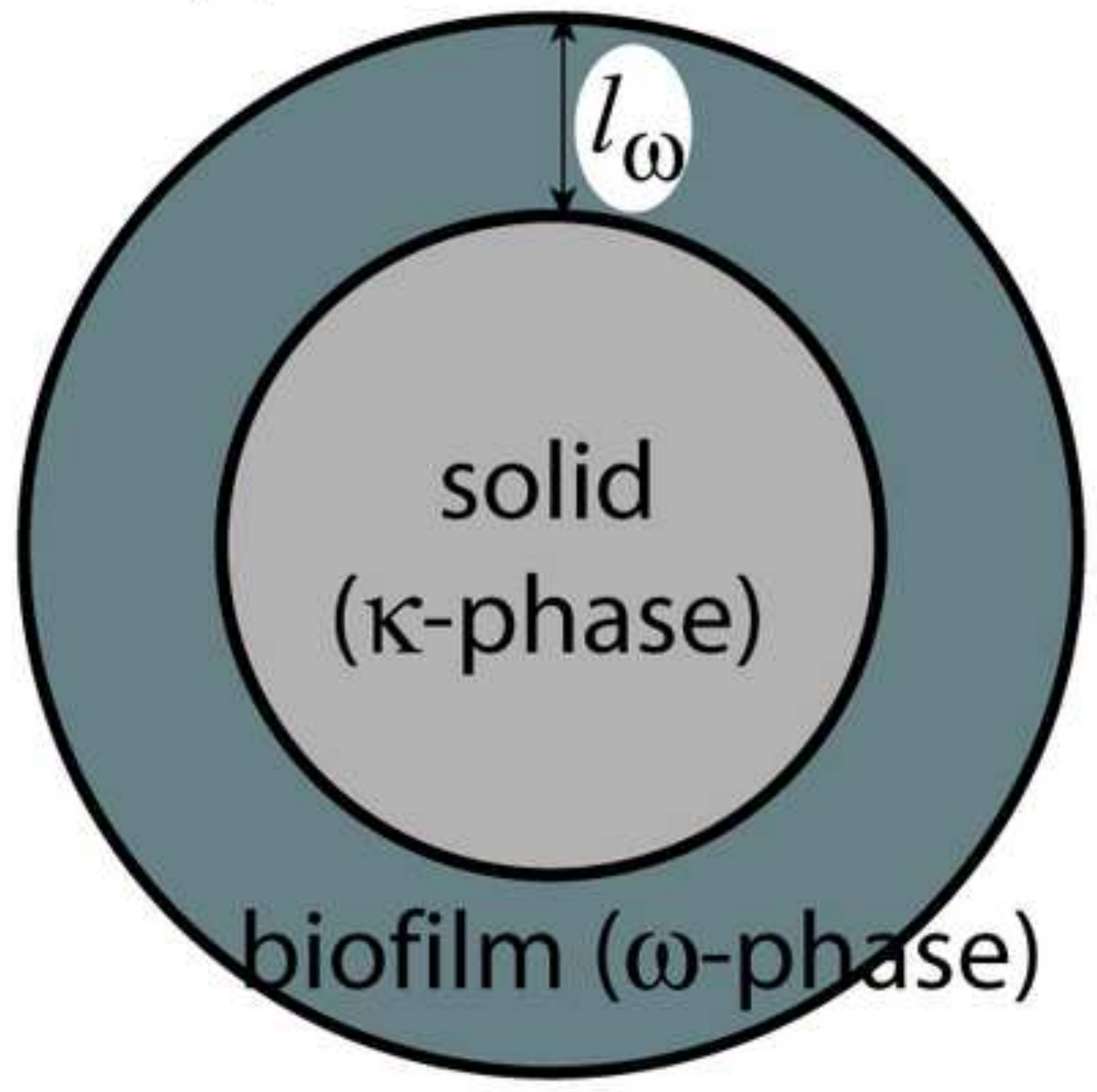

$l_{\gamma}$ 
Concentration

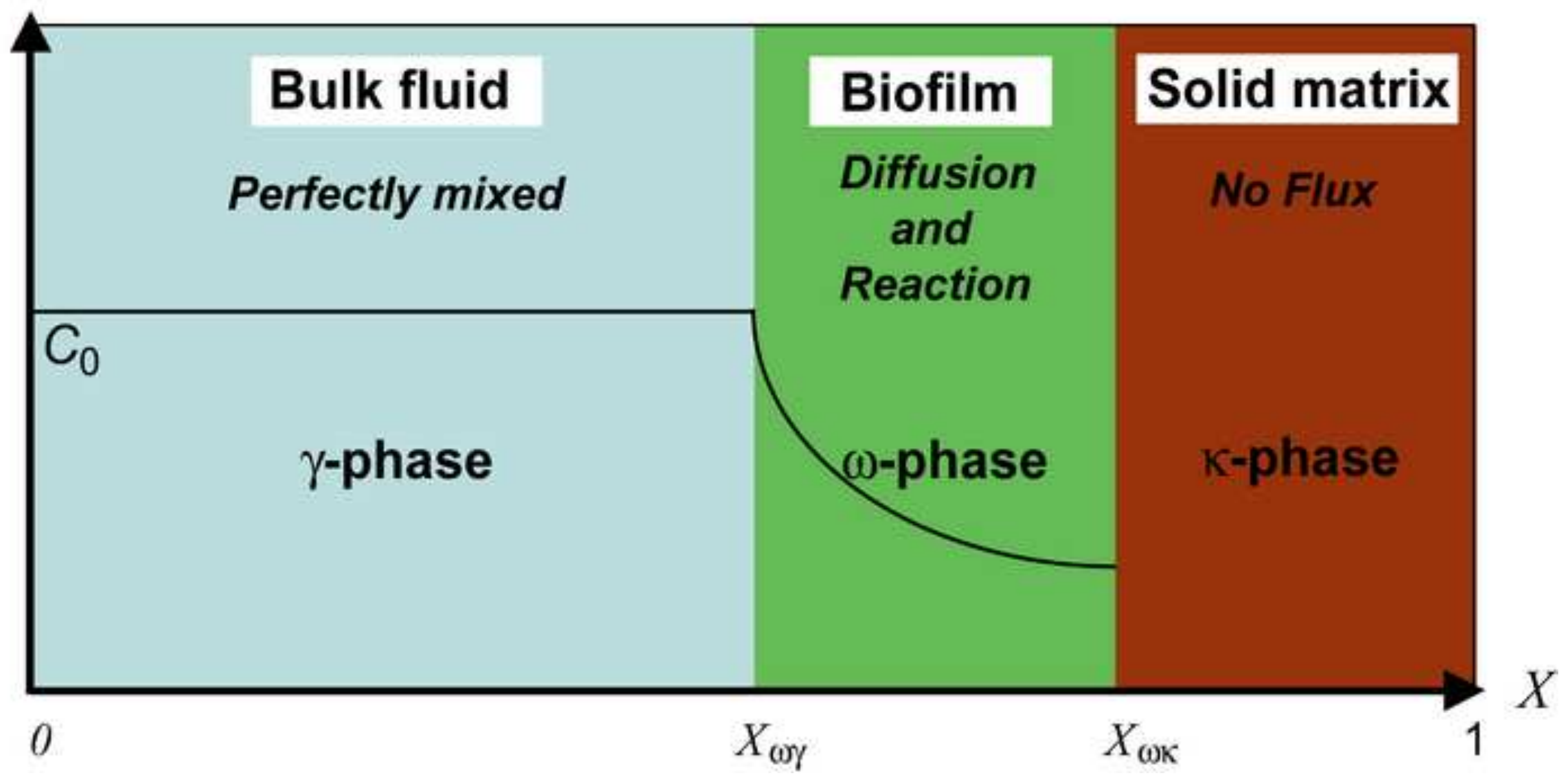


Click here to download high resolution image

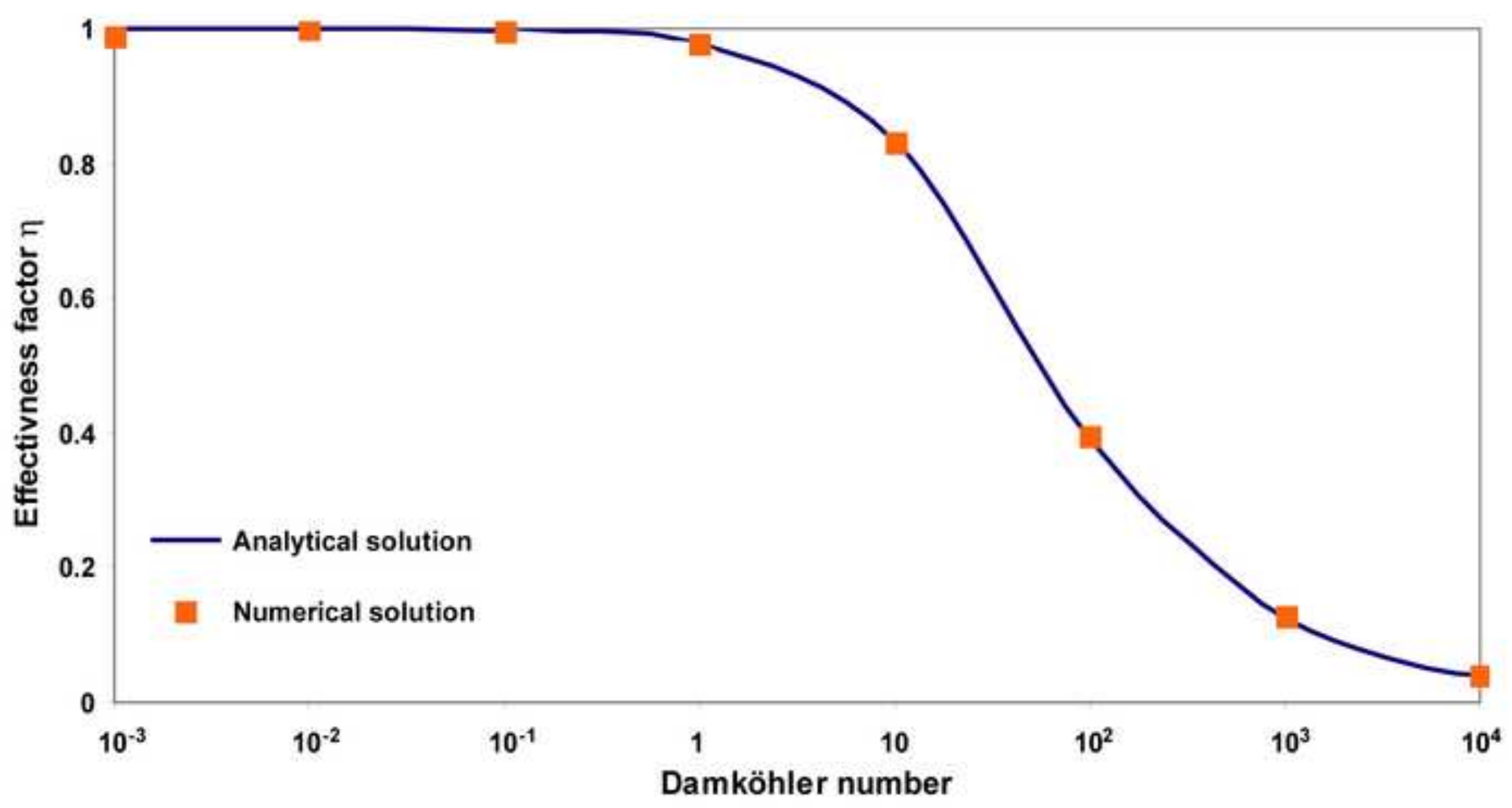




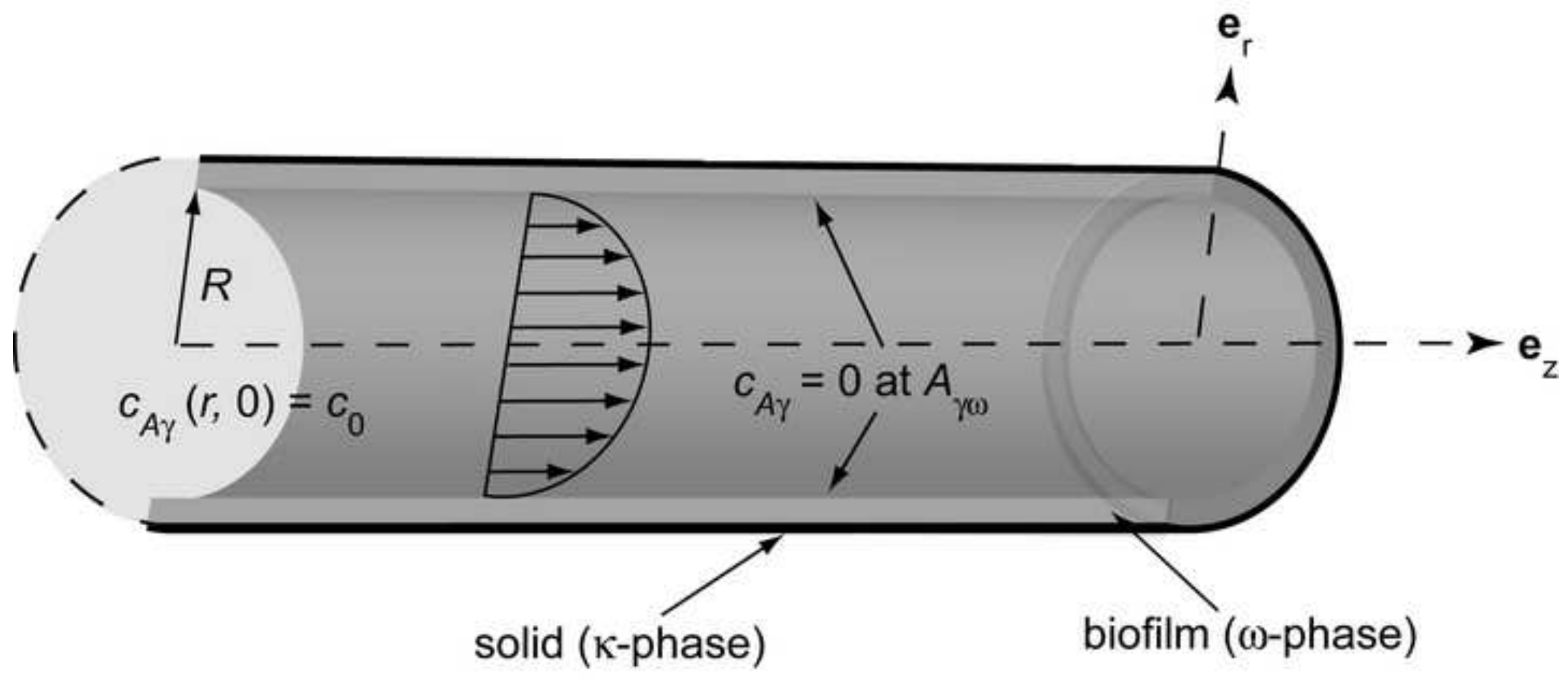


Click here to download high resolution image

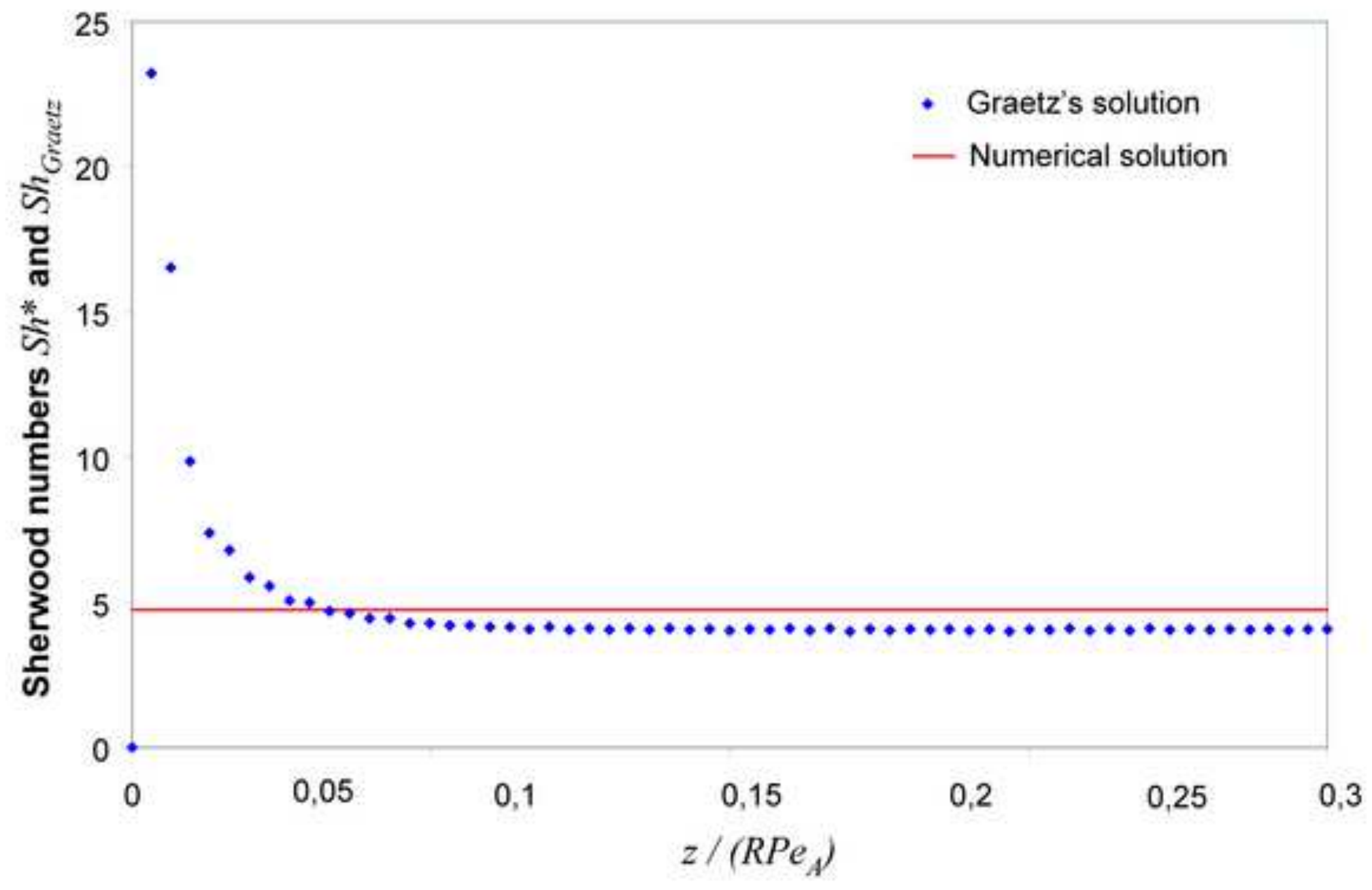




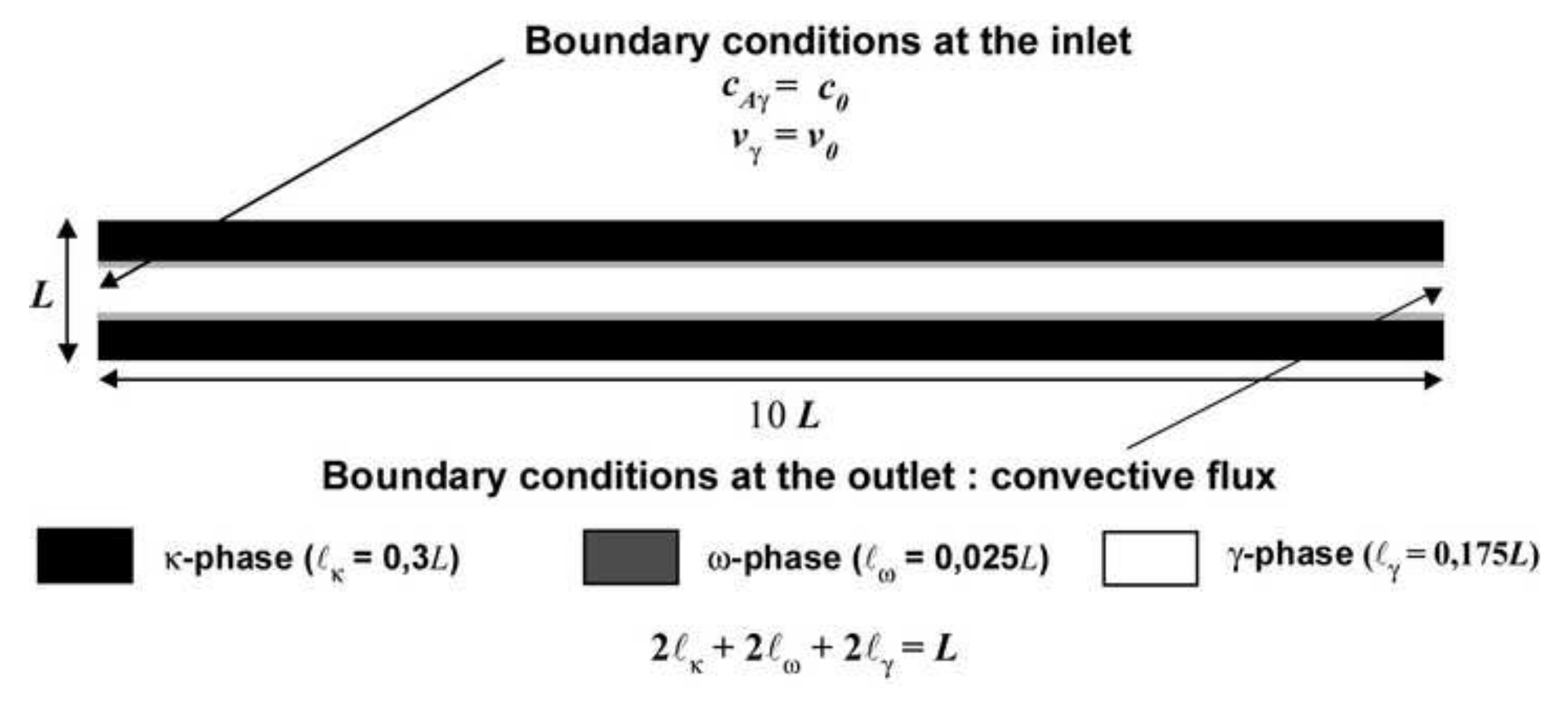

Boundary conditions at the outlet : convective flux

$\kappa$-phase $\left(\ell_{\kappa}=0,3 L\right)$

$$
\begin{gathered}
\square \text { w-phase }\left(\ell_{\omega}=0,025 L\right) \\
2 \ell_{\kappa}+2 \ell_{\omega}+2 \ell_{\gamma}=L
\end{gathered}
$$$$
\square
$$$$
\gamma \text {-phase }\left(\ell_{\gamma}=0,175 L\right)
$$

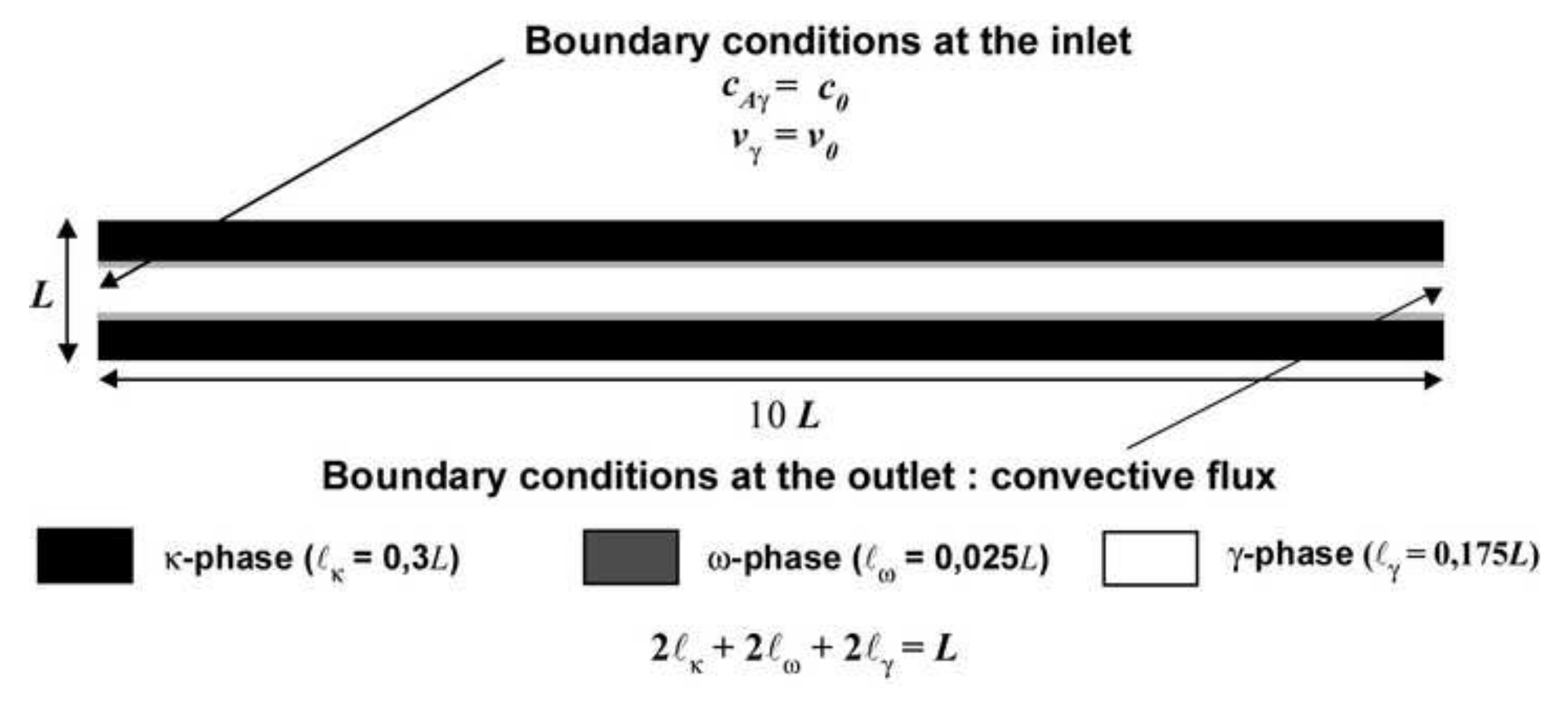$$
\begin{aligned}
& c_{A \gamma}=c_{\theta} \\
& v_{\gamma}=v_{\theta}
\end{aligned}
$$

\section{Boundary conditions at the inlet}

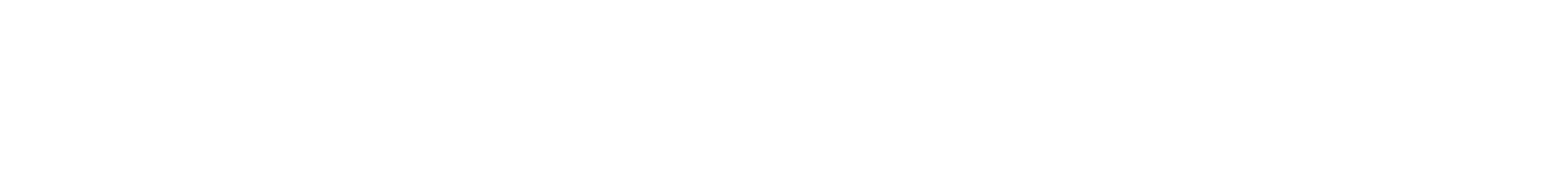




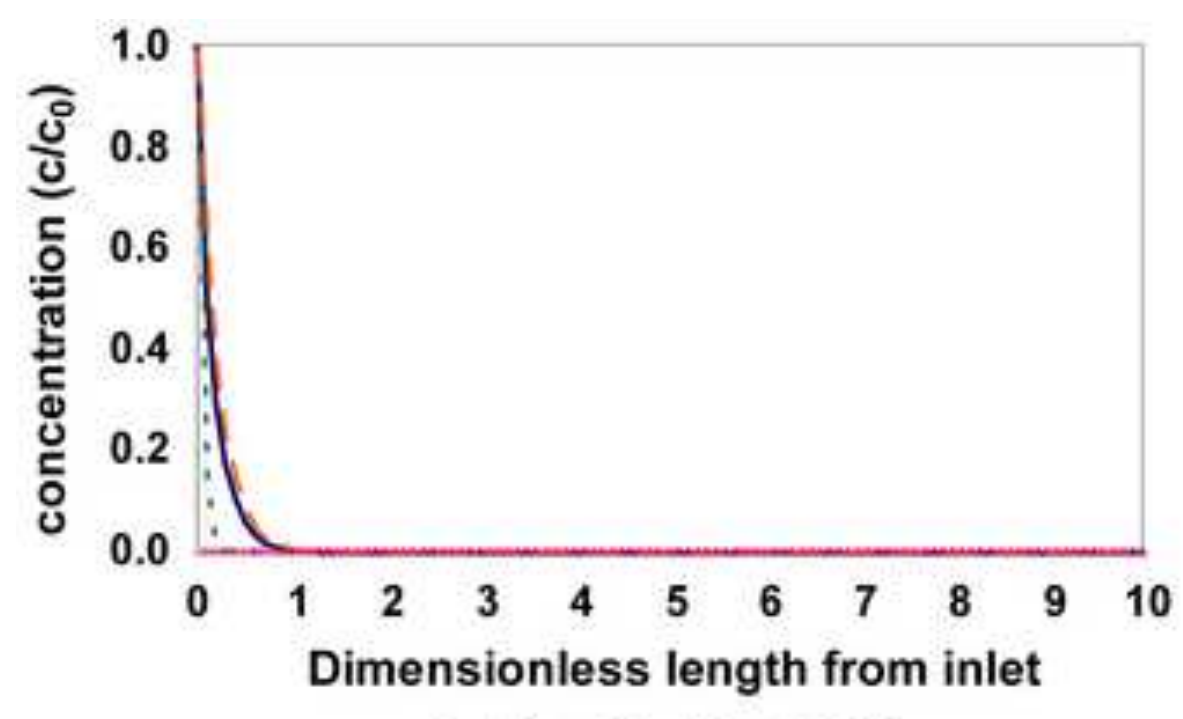

(a) $P e=10 \quad D a=2500$

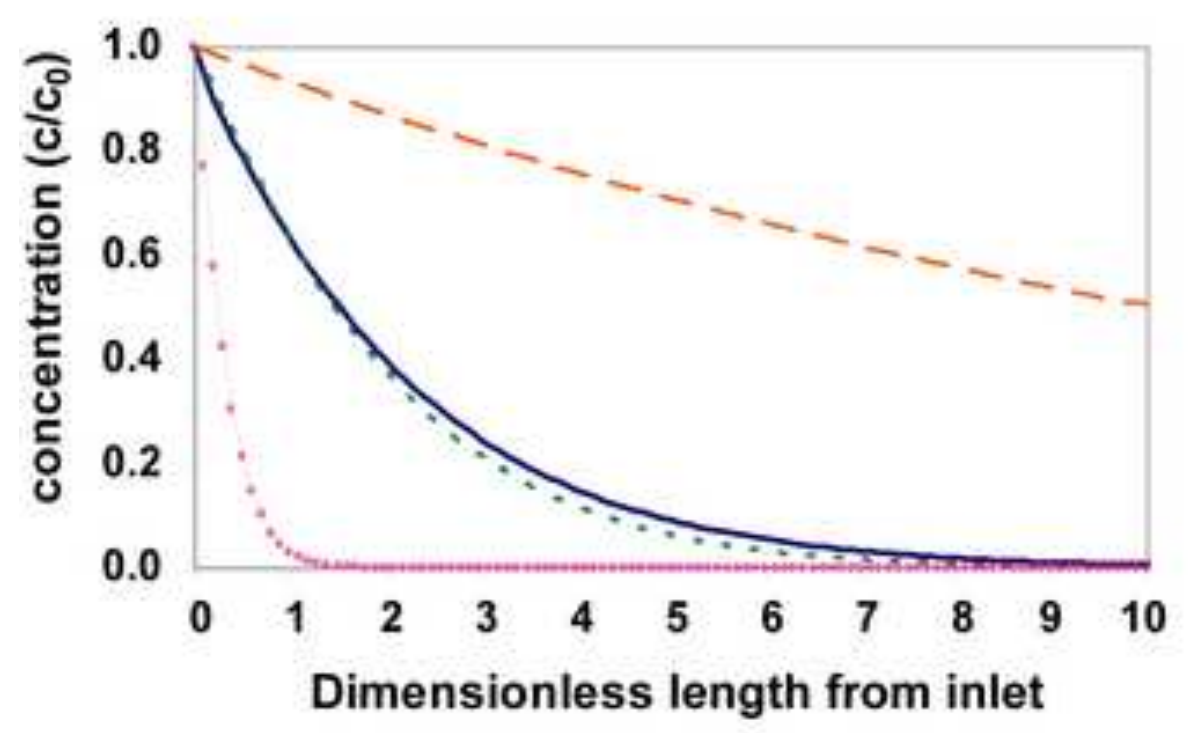

(c) $P e=10^{3} \quad \mathrm{Da}=\mathbf{2 5 0 0}$

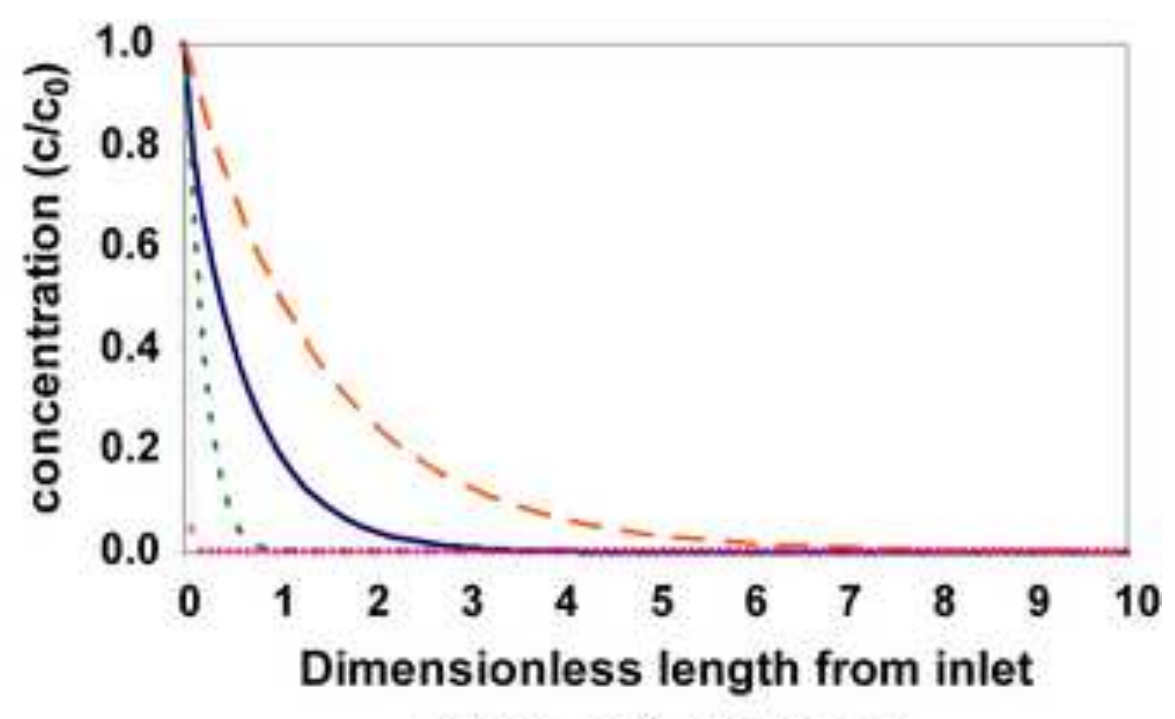

(b) $P e=10^{2} \quad D a=2500$

_ Direct simulation

LEA model

RRLC model

\section{-..- MTLC model}




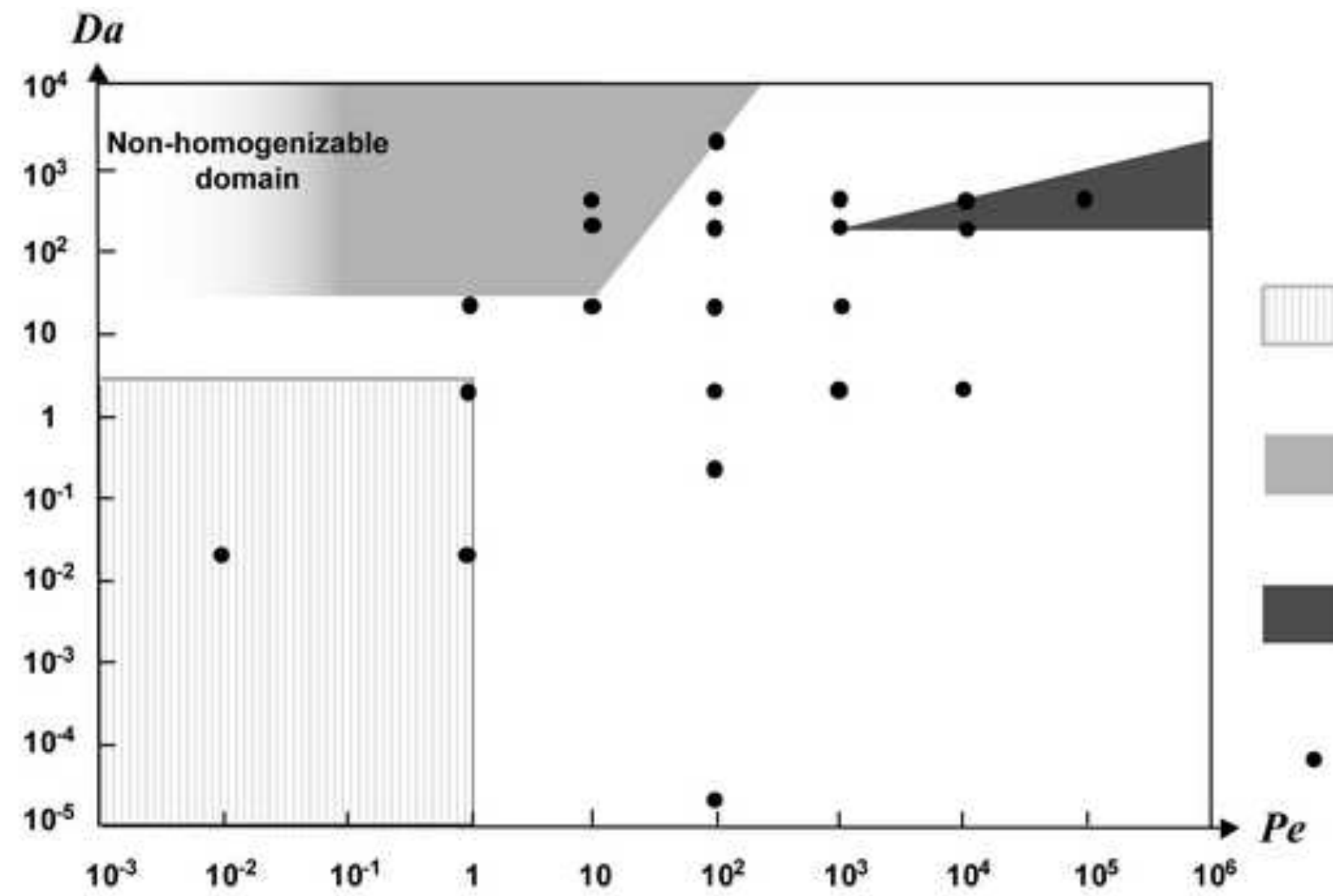

Validity domain of the

LEA model

Validity domain of the

MTLC model

Validity domain of the

RRLC model

Point studied by comparison of direct and averaged simulations 